\title{
LOS RASGOS CULTURALES EN LA CONFORMACIÓN DE LA COMPETITIVIDAD Y DEL CRECIMIENTO SOSTENIBLE
}

\section{CULTURAL VALUES IN THE FORMATION OF COMPETITIVENESS AND SUSTAINABLE GROWTH}

Pavón Cuéllar, Lilianne Isabel (Universidad Anáhuac. México Norte) *

\section{RESUMEN}

El marco de estudio del crecimiento económico consiste en explorar el desempeño de los factores productivos, tanto en calidad como en cantidad, así como la interrelación de estos factores con el producto nacional, todo ello influenciado por el contexto político, social e internacional.

Con el fin de complementar la teoría formal de crecimiento con referentes empíricos y de enfatizar la importancia de considerar elementos adicionales en dicho proceso, esta investigación se concentra en la posible vinculación entre los rasgos culturales de un grupo seleccionado de países y los demás factores productivos, para explorar si dicho capital social informal incide de forma determinante en su conformación y desempeño. Se incluyen también dos variables vinculadas a la equidad y al cuidado medioambiental, al considerarlas insumos esenciales para la competitividad y el crecimiento sostenibles, y se analiza cómo se ven afectadas por la cultura nacional.

Los resultados obtenidos demuestran que la cultura influye en todos ellos a través de distintos canales y que aun cuando esta relación no sea estable en el tiempo o varíe de acuerdo al grado de competitividad alcanzado por un país, su signo positivo o negativo, según el rasgo cultural del que se trate, es consistente entre naciones y en distintas etapas de desarrollo económico.

Palabras claves: Rasgos culturales, competitividad, crecimiento sostenible, factores de producción. JEL: O40, Z10, F01.

\section{ABSTRACT}

The framework of the economic growth study consists in exploring the performance of productive factors, both in quality and quantity, of the interplay of these factors with the domestic product, all influenced by the political, social and international context. In the aim of complementing the formal growth theory with empirical referents and emphasize the importance of considering additional elements in this process, this research focuses on the potential link between the cultural values of a selected group of countries and other production factors, in order to explore whether such informal social capital influences decisively its creation and performance. This work also includes two variables related to equity and environmental care, considered as essential inputs for sustainable competitiveness and growth, as well as the analysis on how these variables are affected by the national culture. Results demonstrate that culture influences all of them through different channels and that even though this relationship is not stable over time or vary according to the degree of competitiveness reached by a country, is consistent across nations and at different stages of economic development.

Facultad de Economía y Negocios, Av. Universidad Anáhuac, núm. 46, Col. Lomas Anáhuac, Huixquilucan, Edo. de México, 52786. lpavon@anahuac.mx

Recibido: Febrero de 2015. Aceptado: Agosto de 2015. 
Key words: Cultural values, competitiveness, sustainable growth, factors of production. JEL: O40, Z10, F01.

\section{INTRODUCCIÓN}

El estudio del crecimiento económico consiste en explorar el desempeño de los factores productivos en su interrelación con el producto nacional, todo ello influenciado por el contexto político, social e internacional. Si el crecimiento viene determinado, entre otros factores, por el capital social de una nación cuya cultura define las reglas del juego o normas de comportamiento formales (marco institucional) e informales (valores y normas sociales), ¿es factible probar la relevancia de la cultura nacional en la competitividad para una muestra representativa de países? Y de ser así, ¿cuáles serían los rasgos que más promueven dicho avance y cuáles serían sus canales de transmisión?. Con el objeto de complementar la teoría formal de crecimiento con referentes empíricos y de destacar la importancia de considerar elementos adicionales en dicho proceso, esta investigación analiza la posible vinculación entre los rasgos culturales de un grupo de noventa y cuatro países y los demás factores productivos, para evaluar si dicho capital social informal incide de forma determinante en su conformación y desempeño. Asimismo, se incluyen dos variables vinculadas a la equidad y al cuidado medioambiental, al considerarlas insumos esenciales para una competitividad y crecimiento sostenibles. Este efecto se da de forma directa, pero también indirectamente a través de la interacción entre las distintas formas de acumulación de capital. Estos efectos se consideran parcialmente al incluir algunas modalidades de capital en la acumulación de otras.

Para probar esta hipótesis general, se evalúan 6 subhipótesis correspondientes al efecto de variables culturales en las distintas modalidades de capital (en sentido amplio): $\mathrm{H}_{\mathrm{o}} \mathrm{a}$ (capital institucional o capital social formal); $\mathrm{H}_{\mathrm{o}} \mathrm{b}$ (sostenibilidad social); $\mathrm{H}_{\mathrm{o}} \mathrm{c}$ (capital humano o educación superior y capacitación); $\mathrm{H}_{\mathrm{o}} \mathrm{d}$ (capital físico o infraestructura); $\mathrm{H}_{\mathrm{o}} \mathrm{e}$ (preservación del capital natural o sustentabilidad medioambiental) y $\mathrm{H}_{\mathrm{o}} \mathrm{f}$ (avance tecnológico $\mathrm{o}$ innovación). En cada una de estas pruebas, se utilizan variables de control para distinguir los efectos de las variables de interés en los distintos factores de crecimiento económico, de otros elementos señalados en la literatura como determinantes de estos últimos.

Los rasgos culturales de un país forman parte de su capital social, pero a su vez inciden y se ven afectados por otras modalidades del capital. Su vinculación con el capital humano es innegable, pues el conjunto de conocimientos, habilidades, competencias y otros atributos incorporados en los individuos, que son relevantes para la actividad económica, son resultado, y al mismo tiempo determinante, del entorno social en el que se adquieren (OCDE, 2003). Asimismo, su relación con el capital físico es evidente, ya que, por ejemplo, la disposición a ahorrar y el espíritu para invertir se encuentran influenciados por los rasgos culturales y viceversa, e incluso el capital natural parece estar íntimamente relacionado con la cultura de un país, tanto en lo que se refiere a la dotación inicial como a su cuidado. Finalmente, dentro del capital social, además de la cultura y de las instituciones formales, debería incluirse la sostenibilidad social, entendida esta como la capacidad de lograr un crecimiento inclusivo o equilibrado socialmente (WEF, 2014). Este capital en sentido amplio se potencia a través de elementos tales como el avance tecnológico o la innovación, incluidos comúnmente en la literatura en el residuo de Solow.

La estructura del estudio es la siguiente: en la primera parte, se revisa brevemente la literatura reciente vinculada al concepto de crecimiento, competitividad y cultura desde una perspectiva económica. Posteriormente, se relacionan algunos rasgos culturales característicos 
de ciertos países, con los principales factores del crecimiento económico sostenible. La investigación intentará profundizar en los que se revelen como más significativos, comprobar su robustez a través de la subdivisión por etapa de desarrollo de la muestra original, para finalmente dar algunas luces sobre los efectos en el desempeño económico que pudieran ser generalizables, sin dejar por ello de considerar los límites de este tipo de análisis. El estudio finaliza con las conclusiones, limitantes, recomendaciones y sugiere líneas futuras de investigación.

\section{EL MARCO DE ESTUDIO DEL CRECIMIENTO ECONÓMICO}

El origen de las teorías de crecimiento se encuentra en los economistas clásicos ${ }^{1}$, que estaban interesados en determinar sus causas y sus consecuencias para el bienestar. Intentaban descubrir las características naturales, demográficas, tecnológicas, políticas, sociales, culturales y morales de una sociedad que tendía a acumular riqueza y mejorar el nivel de vida de la colectividad, y enfatizaban el papel de las instituciones y de las políticas como determinantes del crecimiento y del bienestar (Alzati, 1997). Más tarde, en la década de los setenta del siglo XIX, la revolución marginalista reorientó el análisis a factores subjetivos como la utilidad y a temas como la asignación de recursos y la eficiencia. El estudio de la macroeconomía, y con ella el estudio del crecimiento económico, se delegó a un segundo plano hasta la Gran Depresión y el surgimiento de la teoría keynesiana.

Keynes devolvió el interés en el estudio de la macroeconomía. No obstante, su análisis se orientó a la determinación del ingreso nacional, del nivel general de precios y del empleo en el corto plazo, centrándose en las fluctuaciones económicas y en su estabilización. Con excepción de Marshall, que construyó una teoría formal del crecimiento, no fue sino hasta los años treinta cuando el crecimiento económico volvió a ser del interés de los economistas (Arndt, 1978). Schumpeter (1934) y Clark (1940) reorientaron el análisis económico hacia estos temas, pero fueron los trabajos de Domar (1946) los que establecieron la conexión entre el marco teórico keynesiano y el punto de vista dinámico del crecimiento económico de largo plazo, mediante su énfasis en el ahorro y en la acumulación de capital.

El punto de partida de la teoría moderna de crecimiento económico lo constituyen los trabajos de Solow (1956) y Swan (1956), donde la economía converge a un estado estacionario, y una vez ahí, la acumulación de capital se detiene. En este modelo de rendimientos decrecientes, la única fuente del crecimiento es el progreso tecnológico, lo que lleva a la predicción de convergencia: los países más pobres tendrán mayores incentivos para ahorrar y una tasa de crecimiento más elevada para la misma tasa de inversión, por lo que tenderán a reducir gradualmente la distancia que les separa de los más ricos. La evidencia empírica muestra, sin embargo, fenómenos de crecimiento divergente más o menos transitorios, adversos a esta hipótesis de convergencia (convergencia sigma ${ }^{2}$ ), dando origen a cuestionamientos sobre la validez del modelo neoclásico. En respuesta, surge en defensa del modelo de Solow la hipótesis de convergencia condicional (convergencia beta ${ }^{3}$ ), que depende de que las economías posean ciertos parámetros similares, como las tasas de ahorro, la depreciación del capital, el crecimiento poblacional o la política pública (De la Fuente y Jimeno, 1999).

\footnotetext{
${ }^{1}$ De Adam Smith a David Ricardo.

${ }^{2}$ Se refiere a la velocidad con la que se acerca un país, región o provincia a su estado estacionario.

${ }^{3}$ Se refiere a la dispersión absoluta o relativa en el nivel de ingreso per cápita entre provincias, regiones o países en base logarítmica.
} 
Todos estos elementos han sido factores inspiradores en la búsqueda de alternativas al modelo neoclásico tradicional, dando lugar en años recientes a la literatura de crecimiento endógeno. Los principales atractivos de estos modelos se basan en endogeneizar el progreso técnico (interpretación tradicional del residuo de Solow) y en plantear diferentes hipótesis acerca de cuáles son los determinantes que explican la tasa de crecimiento de un país o región.

En los modelos de crecimiento exógeno, la tasa de progreso tecnológico aparecía como una variable determinada por fuerzas de carácter no económico o al menos no consideradas explícitamente (Kaldor, 1963). Este supuesto resultaba insatisfactorio, considerando que era probable que el ritmo de innovación tecnológica o de sofisticación empresarial en una economía de mercado respondiera a fuerzas económicas, como por ejemplo el potencial de beneficios que genera un nuevo producto o proceso (De la Fuente y Jimeno, 1999).

Existen diversos elementos que influyen en la asignación de recursos al desarrollo del conocimiento (Makarov, 2010). Los más mencionados en la literatura se refieren al apoyo a la investigación científica básica, los incentivos privados a la innovación, investigación y desarrollo (I+D), el aprendizaje derivado de la práctica y las oportunidades disponibles para personas con talento. También son relevantes aspectos como la protección de los derechos de propiedad intelectual y patentes, así como los vínculos entre centros de educación media y superior y el ámbito productivo y de servicios (Romer, 2000).

Aun así, la revisión de los modelos de crecimiento requiere de una exploración permanente, que va más allá de un criterio de viabilidad matemática. Cobra entonces relevancia un análisis de las teorías complementarias sobre el tema, tanto en el ámbito de la economía formal, como son los modelos de crecimiento endógeno y sus extensiones, como las derivadas de la evidencia empírica, en sintonía con la idea de una teoría "magna" del crecimiento al estilo neoclásico. Estas teorías son congruentes, en cierto sentido, con los modelos formales, pues coinciden en afirmar que todo lo que mejora la eficiencia y la productividad de un país también contribuye al crecimiento de largo plazo. Pero además, su análisis enriquece la literatura sobre la convergencia condicional, ya que involucra elementos más allá del avance tecnológico, que se han dejado fuera de los modelos, sea por la falta de disponibilidad de cifras, sea por la complejidad técnica de su tratamiento (De la Fuente y Jimeno, 1999).

A pesar de sus diferencias, todas las teorías y los estudios empíricos coinciden en afirmar que el crecimiento de un país depende de su capacidad de desarrollar y repartir eficientemente el capital. El ahorro y su correcta canalización a una inversión que se traduzca en acumulación de capital de calidad (en sentido amplio) es el motor de la competitividad y del dinamismo económico. La asignatura pendiente es entender en qué consiste ese capital y qué favorece su acumulación, ya que el residuo que aparece recurrentemente en la estimación de todo modelo de crecimiento, evidencia que, por ahora, la concepción tradicional de este factor requiere ampliarse en sus cuatro vertientes: capital físico (cantidad y calidad de infraestructura terrestre, aérea, portuaria y de telecomunicaciones); capital natural (biodiversidad, minerales, energía fósil, termal o hidroeléctrica y calidad del medioambiente, entre otros); capital humano (educación, "know how", capacitación y salud) y capital social (infraestructura social e instituciones en sentido amplio: cultura, tradiciones, leyes y sistema jurídico, pero también sostenibilidad social, basada en la equidad, en la inclusión y en la cohesión social, así como en la fortaleza de la red de seguridad social, formal o informal).

\section{PRODUCTIVIDAD, COMPETITIVIDAD Y CRECIMIENTO SOSTENIBLE}

Productividad, competitividad y crecimiento son conceptos próximos, pero no sinónimos. El crecimiento económico es consecuencia, tal y como lo señaló Solow (1956), de 
una mayor dotación de factores, así como de una mayor productividad en su utilización y eficiencia económica en su aplicación. La noción de productividad se refiere principalmente a la eficiencia tecnológica, esto es, a las empresas, mientras que la de competitividad corresponde más bien a los mercados (WEF, 2014). La productividad constituye un elemento central de la competitividad, pues es determinante en los costes de producción en relación con los de la competencia. Pero el vínculo no es simultáneo ni único, pues la competitividad está también vinculada al marco institucional y social. Hall and Jones (1996) demostraron que el 89 por 100 de la variación del PIB per cápita se atribuye a una mayor productividad.

El concepto de competitividad surge básicamente de dos teorías fundamentales: el modelo de comercio internacional de Adam Smith (1776) y el modelo de la ventaja competitiva de las naciones o Diamante de Porter (1990), que identifica los determinantes de la ventaja nacional competitiva. Aun así, la definición y alcances de la competitividad evolucionan constantemente, sin existir un consenso generalizado sobre su significado. Tampoco hay una metodología internacionalmente aceptada para la construcción de un índice de competitividad, debido a su condición de indicador relativo: la Real Academia Española define competitividad como la capacidad de competir y/o la rivalidad para la consecución de un fin.

Los dos estudios más reconocidos sobre este tema en el ámbito macroeconómico son el Informe de Competitividad Mundial, desarrollado por el Foro Económico Mundial (WEF por sus siglas en inglés ${ }^{4}$ ) y el Anuario de Competitividad Mundial, del Instituto Internacional para el Desarrollo de la Capacidad de Gestión (IMD, por sus siglas en inglés ${ }^{5}$ ), ambos publicados anualmente con información estadística del Banco Mundial, la Organización de Cooperación y Desarrollo Económicos (OCDE), la Organización Mundial del Comercio (OMC), el Banco Interamericano de Desarrollo (BID) y las Naciones Unidas (ONU), entre otros, así como encuestas anuales a empresarios en el ámbito mundial (Benzaquen et al., 2010). Las dos investigaciones utilizan factores de competitividad similares, si bien la vinculación más directa de los indicadores del WEF con la teoría económica ${ }^{6}$, su mayor utilización de datos estadísticos en relación a las encuestas de empresarios, así como un desglose y accesibilidad más amplios, sugirieron utilizarlos como fuente de medición de la competitividad.

El WEF publica anualmente desde 1979 The Global Competitiveness Report, que mide la competitividad de varios países mediante la construcción de un Índice de Competitividad Global (GCI por sus siglas en inglés ${ }^{7}$ ) compuesto de 12 pilares: instituciones, infraestructura, estabilidad macroeconómica, salud y educación primaria, educación superior, eficiencia del mercado de bienes, eficiencia del mercado laboral, sofisticación del mercado financiero, desarrollo tecnológico, tamaño del mercado, sofisticación empresarial e innovación incluidos en tres subíndices correspondientes a requerimientos básicos, promotores de eficiencia y factores de innovación y sofisticación. En su edición 2014-2015, cubre 144 economías, con más de 100 indicadores e incluye por primera vez un indicador de sostenibilidad medioambiental y social, que se incorpora a este trabajo para subrayar que el cuidado del medio ambiente y la equidad, no son simplemente un resultado deseable del crecimiento, sino un insumo indispensable para su sostenibilidad a largo plazo. Ambos aspectos, aun cuando se derivan del concepto de desarrollo sostenible, otorgan una mayor importancia a la

\footnotetext{
${ }^{4}$ World Economic Forum

${ }^{5}$ International Institute for Management Development (IMD)

${ }^{6}$ La teoría del WEF se basa en el modelo de Porter (1990) e IMD aplica una teoría propia, donde los países se rigen conforme a cuatro fuerzas de su entorno competitivo: desempeño económico, eficiencia gubernamental, eficiencia empresarial e infraestructura (Benzaquen et al., 2010).

${ }^{7}$ Global Competitiveness Index.
} 
productividad como motor de prosperidad y crecimiento. La sostenibilidad social son las políticas, instituciones y factores que permiten a todos los miembros de una sociedad acceder a una buena salud, a la participación ciudadana y a la seguridad; y que maximizan su potencial para contribuir y beneficiarse de la prosperidad del país que habitan. Por su parte, la sostenibilidad del medio ambiente son las políticas, instituciones y factores que garantizan una gestión eficiente de recursos para permitir la prosperidad intergeneracional (WEF, 2014).

El desarrollo económico de un país presenta, por lo general una serie de etapas distintivas que el WEF resume en tres, en sintonía con la literatura especializada ${ }^{8}$. En una primera etapa, una economía compite con base en su dotación de factores, básicamente mano de obra no calificada y recursos naturales. Mantener su competitividad requiere de instituciones que funcionen, de una infraestructura adecuada, de un entorno macroeconómico estable y de una fuerza de trabajo sana con educación básica. Conforme el país se hace más competitivo, los salarios aumentan, y la economía avanza a una nueva fase donde el impulso proviene de avances en productividad y calidad de sus productos. En esta etapa, la competitividad se sustenta en niveles más elevados de capital humano, mercados eficientes, la habilidad de apropiarse de los beneficios de las tecnologías existentes, y un gran mercado doméstico o foráneo. Por último, ya que los países pasan a la etapa impulsada por la innovación, a la economía del conocimiento (Edvinsson, 2003 y 2007), los elevados salarios requieren de nuevos productos, únicos o sumamente diferenciados, lo que involucra sofisticados procesos productivos y su constante innovación.

\section{LA IMPORTANCIA DE LA CULTURA}

Si bien hasta el siglo XIX, la inversión sistemática en capital humano fue prácticamente inexistente, la revolución científica que se da a partir de entonces, otorgó mayor importancia al conocimiento y a las habilidades, si bien sólo en relación a sus efectos en la productividad laboral. Como resultado de esta evolución, en el siglo XX, y sobre todo a partir de los años cincuenta y sesenta, surge el concepto del capital humano como factor condicionante primario del crecimiento y del bienestar de un país. Según Becker (1998), los éxitos económicos de los países asiáticos no se explicarían sin una fuerza laboral bien formada, educada, trabajadora y políticamente ordenada. El recurso natural más valioso para esos países fueron sus habitantes. Las cualidades humanas van ganando desde entonces protagonismo en el logro de la prosperidad económica internacional, y actualmente se consideran al menos tan importantes como la disponibilidad de otros factores de producción (Tortella y Núñez, 2014).

El capital humano, entendido como el conjunto de conocimientos, habilidades, competencias y otros atributos incorporados en los individuos, que son relevantes para la actividad económica ${ }^{9}$, constituye un activo intangible con la capacidad de promover la productividad, la innovación, y de mejorar la probabilidad de ser contratado. Se forma a través de distintas vías, que incluyen las instituciones formales de educación, la capacitación y la experiencia, entre otras. Asimismo, el conocimiento, las habilidades, las competencias y otras características se combinan de forma diferente según el individuo y su entorno. De ahí que cualquier estrategia de formación de capital humano requiere reconocer la influencia del contexto social en el que se crea y se utiliza. En el entorno, existen diversos elementos que contribuyen a su formación, tales como el aprendizaje por vías informales y la demanda por distintas habilidades relacionada con la estructura industrial y la cultura organizacional. La

\footnotetext{
${ }^{8}$ Véase Rostow (1960) y Porter (1990), que son los fundamentos teóricos del trabajo del WEF.

${ }^{9}$ Se opta por esta definición de la OCDE (2003), ya que considera los atributos humanos en sentido amplio, pero los limita al mismo tiempo a aquellos que benefician a través de la actividad económica.
} 
infraestructura social de un país, reflejo de su cultura, incluye ciertas características poblacionales que impactan directamente la productividad y otras que afectan las actitudes y competencias que forman parte del capital humano. El crecimiento es entonces resultado, no sólo de la capacidad de sus individuos, sino de su capacidad de acción colectiva. El éxito de las sociedades occidentales avanzadas podría encontrarse en su marco institucional y su evolución permanente, que ha hecho posibles nuevos compromisos entre actores sociales (Diamond, 1998).

El capital social influencia la habilidad de adquirir capital humano a la vez que afecta el entorno en el cual este se desarrolla (Coleman, 1990). A su vez, la formación de capital humano incide en la formación de capital social. Este incluye a las instituciones, definidas por North (1990) como las restricciones diseñadas por los seres humanos que dan forma a la interacción entre individuos, reflejan la cultura de una sociedad y establecen las reglas del juego: normas de comportamiento formales (leyes, regulaciones, contratos y procedimientos internos de ciertas organizaciones) y convenciones informales (cultura, valores o códigos implícitos de conducta), así como las características de aplicación de ambas. Estas actitudes conforman la cultura de un país o región, que Tylor (1924) define como un todo complejo que incluye el conocimiento, las creencias, el arte (incluyendo la tecnología), la moral, el derecho, las costumbres y cualesquiera otros hábitos y capacidades adquiridas por el hombre como miembro de una sociedad. Todas estas características, se transmiten a los individuos a lo largo de su vida y cambian a un ritmo relativamente lento (Becker, 1976).

La complejidad del concepto de cultura ha llevado a los estudiosos a definirla como un conjunto interactivo de características comunes a la población de una nación (Hofstede, 1980, 2003). Según Hofstede (2003), la cultura moldea los esquemas cognitivos del individuo, programando patrones de comportamiento coherentes con su contexto cultural. Está formada de personas que comparten actitudes, valores y creencias, de tal forma que estas pertenecen en forma simultánea a culturas nacionales, étnicas, profesionales y organizacionales. Al mismo tiempo, las actitudes, los valores y las creencias, tanto individuales como grupales, evolucionan, aunque lentamente (Hill, 2009). Definir una cultura nacional no está exento de críticas, pues es factible que exista más de una cultura en un país, sobre todo en un contexto global y de avance en telecomunicaciones. Al mismo tiempo, dicha globalización induce a una creciente interacción que acaba por alterar las culturas y dificulta aislar subgrupos culturales entre naciones (Clark, 1990). Además, hacerlo sería cuestionable, pues cada nación ha adquirido una identidad propia derivada de la fusión cultural. Separar por países pareciera entonces una aproximación aceptable de cultura, o al menos, la más viable. Los cambios culturales suelen darse en un periodo de tiempo relativamente largo (Dawar y Parker, 1994). Como afirma Becker (1996), los individuos tienen menor control sobre su cultura que sobre otras formas de capital social. No pueden alterar su origen étnico ni su historia familiar, y sólo con dificultad pueden cambiar de país o religión. Esta persistencia justifica considerar los rasgos culturales como constantes en nuestro periodo de estudio, o determinados en décadas e incluso siglos anteriores y, por lo tanto, como causal exógeno de la competitividad.

La antropología, la sociología, la historia y la psicología pueden aportar valiosos elementos para evaluar el impacto de los rasgos culturales en la actividad económica (Minkov et al., 2013), ya que las diferencias culturales podrían explicar, por ejemplo, por qué lo que funciona para un país no funciona para otro (Jaén, Fernández-Serrano y Liñán, 2013). De ahí la presunción de que exista una interdependencia importante entre cultura y competitividad. Para evaluar cuantitativamente el impacto cultural en la competitividad de un país, es necesario recurrir a investigaciones que midan estas características internacionalmente en un formato que facilite su procesamiento cuantitativo. Según el catálogo de instrumentos para 
medir la cultura de Taras (2010), existían en 2010 más de 150 instrumentos disponibles. Entre los trabajos interculturales, destacan los trabajos de Hofstede (1980, 2011), Inglehart (1997), Steenkamp (2001), Schwartz (2006) y el proyecto Globe (House, et al., 2004), los de mayor impacto en la literatura especializada (Hsu et al., 2012). Desde un punto de vista estrictamente teórico, los diferentes modelos parecen compartir algunas de las dimensiones integradas en el concepto de cultura nacional, si bien no son pocos los factores que los distancian. Así, cada uno de los estudios mencionados ha utilizado diferentes unidades de análisis, diferentes momentos de tiempo y elaborado con metodologías y muestras poblacionales distintas.

Una comparación entre las distintas investigaciones sugiere que el modelo de Hofstede precisa e identifica más dimensiones culturales, se apega más al marco teórico vigente, se actualiza con mayor frecuencia y amplía constantemente su muestra de países. Quizás ello explique que sea considerado el marco más preciso para el estudio internacional de las culturas nacionales y el más socorrido en la literatura especializada (Hsu et al., 2012). Las similitudes entre sus dimensiones culturales y las distintas tipologías culturales existentes y de publicación posterior (Clark, 1990), así como la validación de sus dimensiones culturales por cerca de 150 estudios internacionales, aun siendo objeto de numerosas críticas ${ }^{10}$, justifican que en esta investigación se opte por este autor para definir la cultura de un grupo de países.

\section{LAS DIMENSIONES CULTURALES SEGÚN HOEFSTEDE}

Hofstede et al. (2010) definen a la cultura como la programación mental que distingue a miembros de un grupo de personas de otros; en el caso de la cultura nacional, dicho colectivo hace referencia a individuos de un mismo país. Consideran que una dimensión cultural es un rasgo que puede medirse en términos relativos y aunque cada dimensión se evalúa con muestras distintas, los puntajes asignados son estables en el tiempo y las perturbaciones que las alteran suelen ser de naturaleza global o al menos continental, de forma tal que al afectar a todas por igual, mantienen inalteradas sus posiciones relativas, salvo casos extremos como una guerra. A continuación se presenta una breve descripción de estas seis dimensiones.

Distancia del poder: contrario al igualitarismo y mide el grado en el que miembros (menos poderosos) de una sociedad aceptan o esperan la existencia de diferencias en los niveles de poder. Una puntuación alta sugiere que la estructura social será más jerárquica e indica, por lo general, que el país es más violento.

Individualismo: se opone al colectivismo y mide el grado en el que los individuos se integran en grupos y poseen un sentido de pertenencia y lealtad a los mismos. Un elevado individualismo refleja vínculos sociales débiles, de tal suerte que el individuo espera valerse por sí mismo.

Masculinidad: se contrapone a femineidad y mide la distribución de roles emocionales tradicionales entre géneros. Las sociedades masculinas son más asertivas y competitivas, con una mayor brecha entre roles y una búsqueda más marcada del éxito material En culturas femeninas, más modestas y empáticas, existe una mayor preferencia por el consenso, la cooperación, el cuidado de los débiles y la calidad de vida.

Intolerancia a la incertidumbre: define la tendencia de una sociedad a evitar la incertidumbre, el riesgo y la ambigüedad. Las sociedades con poco umbral a la incertidumbre son más emocionales y ansiosas, por lo que suelen reforzar la seguridad con leyes estrictas y verdades absolutas a un nivel filosófico y religioso. Son menos tolerantes y reflexivas.

\footnotetext{
${ }^{10}$ Por ejemplo las de Cooper (1982) y McSweeney (2002).
} 
Orientación a largo plazo o pragmatismo: las sociedades orientadas a ella valoran los compromisos a largo plazo, son perseverantes y aceptan la realización de un esfuerzo actual a cambio de una gratificación posterior: muestran una perspectiva pragmática, orientada hacia el futuro. Se contrapone a la consecución inmediata de objetivos y recompensas.

Indulgencia: en contraste con la restricción, simboliza la libertad, la permisibilidad para satisfacer los impulsos humanos básicos y naturales, especialmente los relacionados con disfrutar la vida y el esparcimiento. Su débil correlación negativa con el pragmatismo, hace de esta dimensión un complemento a la anterior (Minkov y Hoefstede, 2011).

\section{CULTURA, COMPETITIVIDAD Y CRECIMIENTO SOSTENIBLE}

En este apartado se vinculan los temas tratados en apartados anteriores, tales como cultura, crecimiento y productividad. En concordancia con la moderna teoría institucional, el capital social afecta y se ve afectado por la situación económica de un país, por lo que no debe establecerse un sentido de causalidad entre estos, sin imponer supuestos arbitrarios a priori. No obstante, como ya se comentaba, para poder estimar el impacto de las instituciones en el desempeño de la economía, es necesario previamente obtener una o varias fuentes de variación exógena de estas, para luego atender a la retroalimentación existente entre instituciones y economía que estudia la Nueva Economía Institucional (NIE) ${ }^{11}$.

North (1990) define al capital social como las normas de comportamiento formales y convenciones más informales, tales como la cultura, valores o códigos implícitos de conducta, así como las características de aplicación de ambas, que han sido moldeadas por la historia de una sociedad, es decir, por fuerzas inmutables a corto plazo (Becker, 1976). Ello justifica considerarlas como variables exógenas en el periodo de análisis y, por ende, analizar su impacto en el desempeño económico de un colectivo. La relevancia de este tipo de estudios es que, puesto que las costumbres persisten o se adaptan gradualmente a las transformaciones de las instituciones formales, pueden surgir discrepancias entre ambos componentes de la estructura institucional. Por consiguiente, incluso si una sociedad imita las instituciones de otra, las diferencias en las reglas informales, pueden explicar los distintos resultados. Como consecuencia, los valores, normas y creencias sociales presumiblemente afectan significativamente los procesos de acumulación de factores y de avance tecnológico, a la vez que determinan la calidad institucional. Existe una amplia literatura que los relaciona ${ }^{12}$ : se estudian por ejemplo, los elementos culturales que inciden en los procesos de producción e inversión, en la innovación tecnológica y en la apertura comercial. También se ha observado que ciertas actitudes afectan directamente al crecimiento, tales como la austeridad, que determina el ahorro e impacta a la inversión en capital físico, o la ambición, que induce a la inversión en conocimiento y a la acumulación de capital humano (Azariadis y Drazen, 1990).

Asimismo, existen otros rasgos sociales, cuyo vínculo con las variables clave del crecimiento económico son menos evidentes, sin que por ello su importancia sea menor (Javidan et al., 1998). Algunos pueden no ser captados por las estadísticas convencionales, ni queda clara su naturaleza, su intensidad, su alcance o sus tiempos. Entre ellos se encuentran los valores morales, decisivos en la productividad. Así por ejemplo, el respeto mutuo y la honestidad son la base de la ética de los negocios y de los derechos de propiedad: su existencia reduce el coste de los contratos y minimiza actividades parasitarias como la búsqueda de rentas. De forma similar, la disposición a cooperar con extraños determina la calidad y magnitud de las redes comerciales, que permiten expandir los mercados nacional e

\footnotetext{
${ }^{11}$ Además de realizar las pruebas de endogeneidad de Haussman pertinentes en estos casos.

${ }^{12}$ Véase por ejemplo Hofstede (1980), o Faria y León Ledesma (2004).
} 
internacionalmente. Por su parte, el grado de apertura social a cuestionamientos o críticas, es decir, la tolerancia, nutre la creatividad, el aprendizaje, la inventiva y facilita la adopción de nuevas tecnologías ${ }^{13}$.

La convergencia condicional vincula al capital humano al concepto de capital social, pues varios de los factores que enumera Barro como variables de control para probar su hipótesis, parecen relacionarse con el entorno. Los rasgos culturales son determinantes en la calidad institucional, en la formación de capital humano y en la eficacia de la política económica. Crecimiento económico, productividad y competitividad son entonces función, por una parte, de la disponibilidad de recursos y de su asignación y, por otro, de factores contextuales que no deben ser subestimados (Barro y Sala-i-Martín, 1995).

Para lograr el objetivo de esta investigación, se identifican los rasgos culturales nacionales con base en la clasificación de Hofstede y se explora si existen relaciones significativas y robustas entre estos y la formación y/o preservación de los factores productivos. Se incluye también la sustentabilidad medioambiental y social. La información económica se extrae del Índice de Competitividad Global del WEF y se inicia el análisis cuantitativo con correlaciones de Pearson entre dimensiones culturales, para proseguir con regresiones por mínimos cuadrados ordinarios de corte transversal. Los índices de competitividad se construyen como un promedio de 2006 a 2015, mientras que los datos culturales de Hofstede utilizados corresponden a información recabada entre 1969 y 2009. Los países que coinciden en ambas muestras son $97^{14}$ si bien en algunas dimensiones e índices de competitividad la muestra fue ligeramente menor ${ }^{15}$. La importancia de iniciar con correlaciones radica en averiguar las relaciones existentes entre dimensiones culturales, ya que de existir un vínculo más estrecho entre ellas que con las variables que se desean explicar, habría que optar por alguna, pues su inclusión en un análisis de regresión provocaría multicolinealidad. Este análisis muestra una correlación negativa moderada entre distancia del poder e individualismo (-0.65) y pragmatismo e indulgencia (-0.44), así como una correlación positiva baja entre individualismo y pragmatismo (0.22). El resto de las dimensiones no parecen estar correlacionadas de forma significativa.

Con base en la revisión de la literatura, es evidente que la cultura de un país incide, no sólo en la formación de un capital social de calidad, sino también en el capital físico, en el capital humano, en el capital natural e incluso en la innovación. De ahí que este trabajo pretenda verificar la hipótesis general de que los rasgos culturales de un país juegan un papel determinante en la formación de capital nacional, entendiéndose este en sentido amplio, con el fin de incluir las cuatro modalidades principales del mismo: capital social, capital físico, capital humano y capital natural. Este efecto se da de forma directa, pero también

\footnotetext{
${ }^{13}$ Véanse los trabajos de Altman (2001) y Blum, Ulrich y Dudley (2001).

${ }^{14}$ Los países incluidos en la muestra fueron: Albania, Alemania, Angola, Arabia Saudita, Argentina, Australia, Austria, Bangladesh, Bélgica, Bután, Brasil, Bulgaria, Burkina Faso, Canadá, Cabo Verde, Chile, China, Colombia, Corea del Sur, Costa Rica, Croacia, Dinamarca, Ecuador, Egipto, El Salvador, Emiratos Árabes Unidos, Estados Unidos, Eslovaquia, Eslovenia, España, Estonia, Etiopia, Filipinas, Finlandia, Francia, Ghana, Grecia, Guatemala, Honduras, Hong Kong, Hungría, India, Indonesia, Irán, Irlanda, Islandia, Israel, Italia, Jamaica, Japón, Jordania, Kenia, Kuwait, Letonia, Líbano, Libia, Lituania, Luxemburgo, Malawi, Malasia, Malta, Marruecos, México, Mozambique, Namibia, Nepal, Nueva Zelanda, Nigeria, Noruega, Países Bajos, Pakistán, Panamá, Perú, Polonia, Portugal, Reino Unido, República Checa, República Dominicana, Rumania, Rusia, Senegal, Serbia, Sierra Leona, Singapur, Sudáfrica, Surinam, Suecia, Suiza, Taiwán, Tanzania, Tailandia, Trinidad y Tobago, Turquía, Uruguay, Venezuela, Vietnam y Zambia.

${ }^{15}$ Si no había información de la competitividad para todo el periodo en un país, se utilizaba el promedio de los años disponibles. De no contar con información sobre un rasgo cultural o índice de competitividad, se omitía solo en las regresiones que lo incluyeran. Este ejercicio permitió también solucionar problemas de observaciones atípicas, pues los países descartados eran los que las presentaban, evitando formas funcionales incorrectas.
} 
indirectamente a través de la interacción entre sus distintas formas ${ }^{16}$. Para probar esta hipótesis general, se evalúan 6 subhipótesis, que incluyen también variables de control, para distinguir los efectos de las variables culturales en los distintos motores de crecimiento económico, de otros elementos señalados en la literatura como determinantes de estos.

Hoa: el capital social informal, bajo la forma de los rasgos culturales medidos por Hofstede como la distancia del poder, el individualismo, la masculinidad, la tolerancia a la incertidumbre, el pragmatismo y/o la indulgencia, incide en la formación de capital físico (infraestructura) del país.

Hob: el capital social informal, bajo la forma de los rasgos culturales medidos por Hofstede como la distancia del poder, el individualismo, la masculinidad, la tolerancia a la incertidumbre, el pragmatismo y/o la indulgencia, incide en la formación de capital humano (educación superior y capacitación) del país.

Hoc: el capital social informal, bajo la forma de los rasgos culturales medidos por Hofstede como la distancia del poder, el individualismo, la masculinidad, la tolerancia a la incertidumbre, el pragmatismo y/o la indulgencia, incide en la calidad institucional (capital social formal) del país.

Hod: el capital social informal, bajo la forma de los rasgos culturales medidos por Hofstede como la distancia del poder, el individualismo, la masculinidad, la tolerancia a la incertidumbre, el pragmatismo y/o la indulgencia, incide en la sostenibilidad social del país.

Hoe: el capital social informal, bajo la forma de los rasgos culturales medidos por Hofstede como la distancia del poder, el individualismo, la masculinidad, la tolerancia a la incertidumbre, el pragmatismo y/o la indulgencia, incide en la preservación del capital natural del país.

Hof: el capital social informal, bajo la forma de los rasgos culturales medidos por Hofstede como la distancia del poder, el individualismo, la masculinidad, la tolerancia a la incertidumbre, el pragmatismo y/o la indulgencia, incide en el avance tecnológico (innovación) del país.

La persistencia de los rasgos culturales, comentada con anterioridad en este trabajo, sugiere que el periodo de estudio de la competitividad ( 9 años), garantiza que cualquier efecto temporal que pudiera llevar a dobles causalidades sea marginal, pero aun si dicho efecto existiese, sería neutralizado por el desfase entre los periodos de evaluación de las variables explicativas y la explicada, así como por la naturaleza transversal de los datos. Adicionalmente, sólo se busca establecer si existe un efecto significativo de los rasgos culturales nacionales en la competitividad de un país y su signo, y no una relación cuantitativa exacta con fines de inferencia estadística.

Aun cuando sólo se incluyen las finalmente elegidas, se estimaron múltiples regresiones para lograr un mejor ajuste, comprobar que las relaciones encontradas fueran robustas, con signos consistentes y excluyendo aquellas variables que pudieran dar lugar a multicolinealidad por su relación con otras. También se depuraron para quedarse con aquellas cuyos estimadores cumplieran cabalmente con las propiedades necesarias para extraer

\footnotetext{
${ }^{16}$ Estos efectos se consideran parcialmente al incluir algunas modalidades de capital en la acumulación de otras.
} 
información confiable sobre las posibles vinculaciones entre cultura y formación de capital ${ }^{17}$. Debido a que la correlación entre distancia del poder e individualismo fue mayor, en valor absoluto, a la de estas dimensiones con cualquiera de las variables dependientes utilizadas, se consideró pertinente que el modelo finalmente elegido excluyera a una de las dos, aun cuando Franke, Hoefstede y Bond (1991) han planteado que pueden incluirse ambas. Si bien no es evidente la razón de considerar como sustitutos al colectivismo y a la distancia del poder, es claro que una sociedad poco individualista, es más propensa a aceptar la sumisión implícita en una estructura social jerárquica que obliga a asumir que ciertas personas gozan de mucho mayor poder que otras. Como afirma Schwartz (2006), el individualismo alienta la autonomía y dota de empoderamiento a las personas para preocuparse por sus propias necesidades. En el caso de las otras dos dimensiones culturales relacionadas, pragmatismo e indulgencia, se decidió incluirlas, si bien cuidando que no causaran problemas en la regresión, pues en ese caso se eliminaría la que aportara menos al poder explicativo del modelo. En general, las regresiones presentaron una bondad de ajuste de buena a moderada y sus variables explicativas resultaron ser altamente significativas ${ }^{18}$. En el cuadro 1 se presentan con mayor detalle las finalmente elegidas, para luego continuar con las observaciones que emanan de ellas.

De acuerdo a $\mathbf{H}_{\mathbf{0}} \mathbf{a}$, la formación de capital físico depende positivamente del pragmatismo y de la indulgencia, una vez incorporado el efecto del desarrollo de los mercados financieros, la coyuntura económica ${ }^{19}$ y el tamaño del mercado ${ }^{20}$. La inversión en infraestructura se dispara en la fase expansiva del ciclo de negocios cuando no existen restricciones de liquidez relacionadas con una penetración financiera insuficiente. Asimismo, interconectar el territorio nacional con criterios de evaluación social de proyectos es más factible en sociedades indulgentes que poseen además una visión de largo plazo. Finalmente, un mayor mercado propicia la acumulación de factores y el crecimiento, pues da lugar a economías de escala y de ámbito en su conformación.

$\mathbf{H}_{\mathbf{0}} \mathbf{b}$ sugiere que la formación de capital humano, bajo la forma de educación superior y capacitación, se ve influenciada positivamente por el individualismo, ya considerando el tamaño del mercado y una vez cubierta la educación primaria y los requerimientos básicos de salud de una nación. El tamaño de mercado se constituye una vez más como una condición para el crecimiento y la competitividad, tal y como lo indican Hoefstede y Bond (1990, p.18)): "la cultura (...) es una condición necesaria para el crecimiento económico: no obstante, por sí misma no es suficiente para que dicho crecimiento ocurra. Como mínimo, dos condiciones necesarias adicionales son la existencia de un mercado y un contexto político que permita el desarrollo".

\footnotetext{
${ }^{17}$ Se eligieron las variables con mayor poder explicativo según los criterios de bondad de ajuste $F, R^{2}$ y $R^{2}$ ajustado), así como los que minimizaran los criterios de Akaike, Schwarz y Hannan-Quinn. En cuanto a las propiedades de los estimadores, todas las regresiones lograron normalidad en la distribución de residuos y no hubo indicios de multicolinealidad (ninguno de los coeficientes de correlación entre variables independientes fue cercano a uno (o menos uno) ni mayor al coeficiente de determinación del modelo estimado, salvo individualismo y distancia de poder que siempre se incluyeron por separado.

${ }^{18}$ Se comprobó también que la forma funcional de las regresiones fuera la correcta y que no hubiera variables omitidas o redundantes. Finalmente, se evaluó la posible existencia de heterocedasticidad en el modelo.

${ }^{19}$ Balance presupuestal del sector público como porcentaje del PIB, ahorro nacional, inflación anual, deuda pública como porcentaje del PIB y calificación de crédito (WEF, 2014).

${ }^{20}$ Tamaño del mercado (doméstico y foráneo), PIB ajustado por paridad de poder de compra y exportaciones como porcentaje del PIB (WEF, 2014).
} 
CUADRO 1: PRUEBA DE LAS HIPÓTESIS PARA LA MUESTRA COMPLETA DE PAÍSES

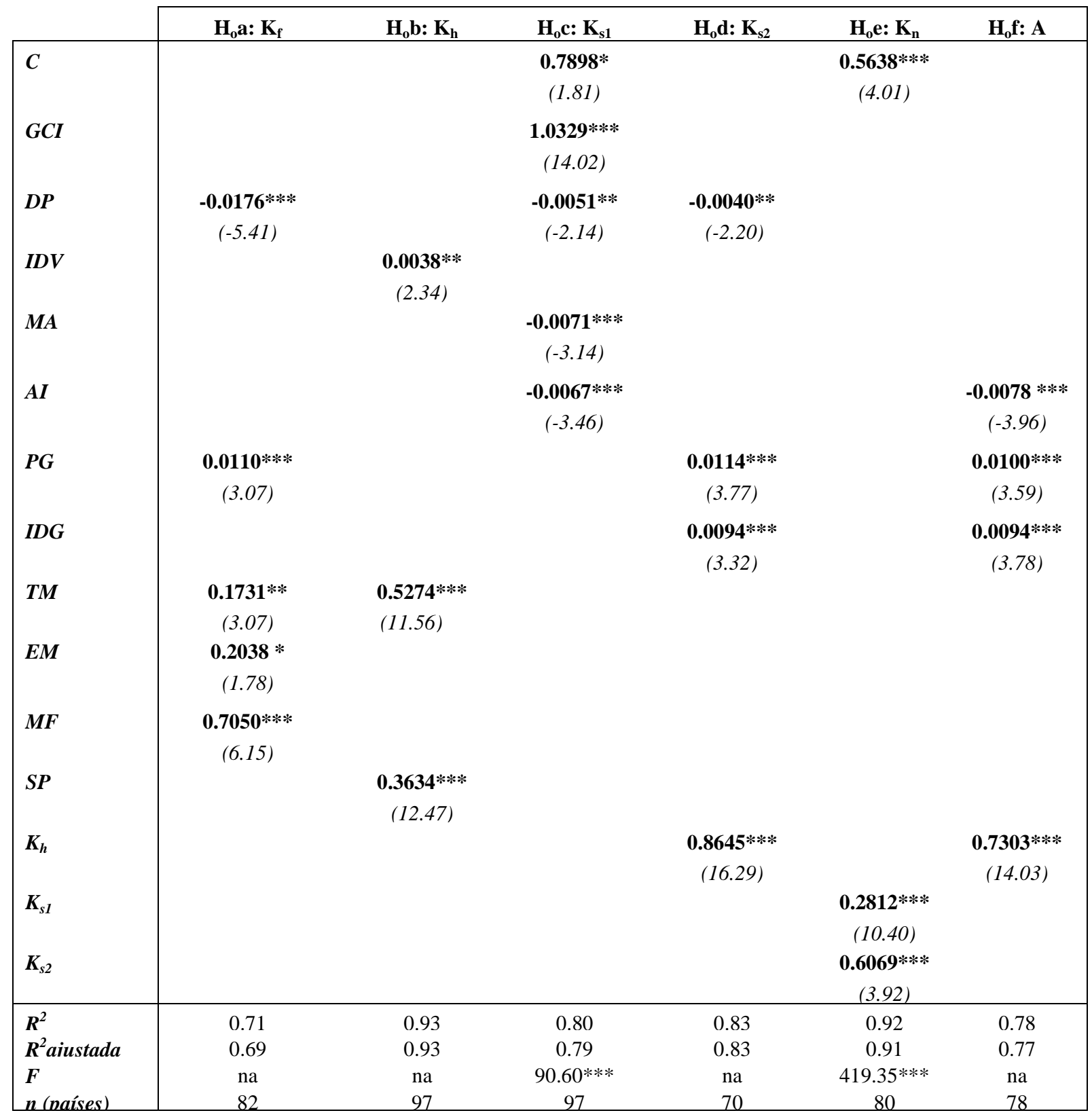

Fuente: Elaboración propia con cifras de WEF (2014), Hofstede (1980, 1991, 1993, 2002 y 2008), Hofstede y Bond (1990), Hofstede y Arrindell (1998), Hofstede et al (2008) y Behbudi et al (2010). na: no aplica

Nota: Los estadísticos t se reportan entre paréntesis: ***,*** denotan significancia a un nivel de 1\%,5\% y 10\%, respectivamente. Dónde:

$\begin{array}{lll}\mathrm{K}_{\mathrm{s} 1} & : & \begin{array}{l}\text { Instituciones (capital social formal) } \\ \mathrm{K}_{\mathrm{h}}\end{array} \\ & : & \begin{array}{l}\text { Educación superior } \mathrm{y} \text { capacitación } \\ \text { humano) }\end{array} \\ \mathrm{K}_{\mathrm{f}} & : & \text { Infraestructura (capital físico) } \\ \mathrm{IDV} & : & \text { Individualismo } \\ \mathrm{PG} & : & \text { Pragmatismo } \\ \mathrm{EM} & : & \text { Entorno macroeconómico (coyuntura) } \\ \mathrm{MA} & : & \text { Masculinidad } \\ \mathrm{TM} & : & \text { Tamaño del mercado } \\ \mathrm{SP} & : & \text { Salud y educación primaria } \\ \mathrm{ML} & & \text { Desarrollo de los mercados laborales } \\ \mathrm{AT} & : & \text { Alfabetización tecnológica }\end{array}$

$\begin{array}{lll}\mathrm{K}_{\mathrm{s} 2} & : & \text { Sustentabilidad social (capital social) } \\ \mathrm{K}_{\mathrm{n}} & : & \text { Capital natural (sustentabilidad medioambiental) } \\ & & \\ \mathrm{DP} & : & \text { Distancia del poder } \\ \mathrm{A} & : & \text { Innovación (Avance tecnológico) } \\ \mathrm{IDG} & : & \text { Indulgencia } \\ \mathrm{AI} & : & \text { Aversión a la incertidumbre } \\ \mathrm{MF} & : & \text { Desarrollo de los mercados financieros } \\ \mathrm{GCI} & : & \text { Competitividad global } \\ \mathrm{RQ} & : & \text { Requerimientos básicos } \\ \mathrm{C} & : & \text { Constante }\end{array}$


El capital social formal de un país, representado en $\mathbf{H}_{\mathbf{0}} \mathbf{c}$ por sus instituciones, depende positivamente de su competitividad, lo que demuestra que los países ricos pueden permitirse mejores instituciones, y no sólo que mejores instituciones dan lugar a países más ricos. Al mismo tiempo, se ve afectado negativamente por la distancia del poder, la masculinidad y la aversión a la incertidumbre, resultados que coinciden con los de Lynn (1991). Esta hipótesis busca cubrir parcialmente la principal limitante de esta investigación, que tiene que ver con el carácter unidireccional del vínculo entre capital social informal y acumulación de capital. La ecuación mantiene esta condición, ya que sólo considera el capital social formal, pero demuestra, conforme a la Nueva Economía Institucional, cuyo campo de estudio queda fuera de los alcances de este trabajo, que el capital social de un país y sus efectos deben también evaluarse desde una perspectiva dinámica. Inglehart (1997) identifica un cambio intergeneracional en los valores culturales de naciones industrializadas, mientras que Inglehart y Welzel (2005) encuentran que el crecimiento económico trae consigo alteraciones en la escala de valores de los ciudadanos, que exigirán mayores libertades democráticas.

$\mathbf{H}_{\mathbf{0}} \mathbf{d}$ sugiere que la sustentabilidad social se promueve en naciones bien educadas con una elevada inversión en capital humano, pero también son importantes rasgos culturales como la indulgencia y el pragmatismo para inducirla, así como la distancia del poder lo es para inhibirla. La equidad, la inclusión y una red de seguridad social que evite el lastre de la pobreza extrema, requieren de cualidades humanas como la indulgencia y la empatía para establecerse, pero también de una visión de largo plazo, de una administración eficiente de recursos escasos así como de una movilidad social que sólo una sociedad educada puede mantener. En sociedades autoritarias, es más factible que existan diferencias de oportunidades y una mayor exclusión social.

La sustentabilidad medioambiental no parece depender directamente de ningún rasgo social en concreto según $\mathbf{H}_{\mathbf{0}} \mathbf{e}$. Quizás por sus características de bien público, que lo hacen no rival y no excluible, sufra de la tragedia de los comunes en una sociedad donde priva la competencia por los recursos. Las regresiones estimadas sugieren que el cuidado medio ambiental sólo podrá ser logrado mediante la existencia de instituciones sólidas, pero sobre todo por una sociedad más justa donde prevalezca la sustentabilidad social. La riqueza del país, la eficiencia de sus mercados reales y monetarios e incluso el tamaño de los mismos no resultaron significativos.

Finalmente, conforme a $\mathbf{H}_{\mathbf{0}} \mathbf{f}$, el avance tecnológico y la innovación ${ }^{21}$ dependen, dada la formación de capital humano del país, de rasgos culturales como la indulgencia y el pragmatismo para impulsarla, y de la aversión a la incertidumbre para frenarla, independientemente de la alfabetización tecnológica de un país o de la eficiencia de los mercados reales o monetarios, que no parecen tener un efecto significativo en ellos. Asimismo, aun cuando la literatura ha demostrado una relación positiva entre individualismo y creatividad, no encuentra una relación confiable entre innovación y distancia del poder, de igual forma que en la presente investigación, y a pesar de que creatividad e innovación son conceptos estrechamente vinculados entre sí. La forma de conciliar ambos resultados es destacar que existen algunas diferencias entre ambos términos, tal y como lo señalan Shane (1993) y Rinne et al. (2012). La distancia del poder se relaciona con aquella parte de la innovación que no se vincula con la creatividad, la fase en la que el inventor debe "salir del laboratorio" y lidiar con las instituciones para registrar, patentar y fondear o vender sus investigaciones. Las dependencias gubernamentales altamente burocráticas y regidas por

\footnotetext{
21 Capacidad de innovación, calidad institucional en I+D, gasto empresarial en I+D, colaboración de universidades e industria en $\mathrm{I}+\mathrm{D}$, proveeduría gubernamental de alta tecnología, disponibilidad de científicos e ingenieros y solicitudes de patentes (WEF, 2014).
} 
largos y rígidos procesos, difícilmente darán cabida a nuevas ideas que no encajen en el statu $q u o$, lo que reduce la probabilidad de que una idea creativa logre acceder al mercado ${ }^{22}$.

Analizando los resultados bajo una perspectiva de rasgos culturales, todos ellos presentaron los signos esperados y fueron significativos en la formación de alguna modalidad de capital o en el avance tecnológico de los países.

En cuanto al pragmatismo u orientación de largo plazo, tal y como lo planteaban Hoefstede y Bond (1990), resulta evidente su efecto positivo y significativo en la formación de capital físico, en la innovación y en la sustentabilidad social. Este rasgo se relaciona con la frugalidad y el ahorro, con el crecimiento y la flexibilidad, a la vez que contribuye a buenos resultados escolares en las comparaciones internacionales y no se lleva bien con el patriotismo (Hoefstede, 2001). Hoefstede y Bond (1990) señalan que este rasgo cultural, característico de países de extremo oriente, conlleva un pensamiento estratégico y un dinamismo que facilita la actividad empresarial, lo que aunado a la importancia del honor en esta cultura, promueve la empatía y el respeto en los intercambios sociales y de negocios, así como la persistencia contribuye al logro de los objetivos, incluidos los económicos. El continuo movimiento característico de esta cultura facilita la absorción tecnológica y la creación y transmisión del conocimiento (Lin y Edvinson, 2011). No obstante, la firmeza de metas, si se lleva al extremo, desalentaría la iniciativa, la toma de riesgo y la flexibilidad necesarios para los emprendedores que intentan explotar las oportunidades de negocio. Por ello, la interrelación cultural puede ser beneficiosa. Los tigres asiáticos han sido ejemplo de estas sinergias culturales, al combinar en la absorción tecnológica estos rasgos orientales con la tolerancia a la incertidumbre y la flexibilidad, rasgos más característicos de occidente.

En lo referente al individualismo, su efecto positivo en el desempeño económico ha sido demostrado por diversos autores, aunque no con la consistencia que se observa en el caso del pragmatismo. En este estudio tiene un impacto significativo en la formación de capital humano, en línea con los resultados de Ros y Gouveia (2003), que encuentran una elevada correlación negativa entre tasa de analfabetismo e individualismo, mientras que Yeganeh (2013) demuestra que la autonomía promueve la competitividad nacional. Hoefstede (2010) ratifica a la autonomía, a la independencia y a la libertad, valores asociados al individualismo, como los rasgos culturales necesarios para promover la creatividad y la movilidad social. En sociedades colectivistas, hay códigos de pertenencia al grupo, por lo que plantear nuevas ideas puede ser considerado como una afrenta a su cohesión. Asimismo, mientras que en sociedades colectivistas el objetivo es aprender a hacer, en sociedades individualistas el sistema educativo se centra en enseñar a aprender, lo que fomenta la creatividad (Gardner, 1989). El individualismo también se relaciona con la riqueza, si bien de forma decreciente conforme mejora la situación de un país, y con lazos familiares débiles y estrechos (Hoefstede, 2001).

La distancia del poder favorece la corrupción, el nepotismo e incluso las mafias, que surgen de fidelidades a la autoridad por encima de la lealtad a los propios valores. Quizás por ello se vincula de forma fuerte y negativa con el individualismo, lo que lleva a que sea un inhibidor importante de calidad institucional. En el mismo sentido, la distancia del poder conlleva a la existencia de jerarquías, donde los subordinados esperan recibir instrucciones sobre lo que debe o no hacerse, en vez de potenciarse la iniciativa y la creatividad. Esto genera una actitud pasiva y parasitaria en la sociedad que lleva a una formación insuficiente de infraestructura. Esta actitud inicia desde el núcleo familiar, el salón de clases y

\footnotetext{
${ }^{22}$ Chatterji et al., en 1993, por ejemplo, argumentan que los sistemas políticos liberales favorecen el libre intercambio y la propagación de ideas que promueven la innovación y la conformación de capital intelectual.
} 
posteriormente en la vida pública, y se traduce en regímenes políticos poco democráticos, élites de poder en el sector privado y público y, en ocasiones, en corrupción y tráfico de influencias al no requerir del consenso y de la transparencia de sociedades más igualitarias. El acceso a la educación y la distribución del ingreso suelen ser desiguales, la movilidad social limitada y el racismo emerge con mayor facilidad. Estos resultados se verifican en este trabajo, ya que este rasgo cultural impacta negativa y significativamente a la sustentabilidad social de un país.

Esta investigación también encuentra que la intolerancia a la incertidumbre está relacionada negativamente con la calidad institucional y con la innovación. El principal motor detrás de esta dimensión de Hofstede parece ser un deseo de control, que puede obstaculizar la creatividad medida a nivel nacional. En su estudio de 1993, Shane no encontró relación entre el número de patentes y la intolerancia a la incertidumbre, si bien sí la halló en 1993 entre esta y los usuarios de dichos inventos: las marcas comerciales. Por ello, llegó a la conclusión de que las culturas intolerantes a la incertidumbre eran más lentas en relación con la innovación, lo que inhibía la competitividad, aun cuando la relación observada entre ellas podía ser positiva o no significativa en la fase de investigación (patentes). Como lo señalan Labra y Sanchez (2013), la economía del conocimiento es el resultado de la creación y el intercambio de ideas, donde las tecnologías de la información y la comunicación son un elemento clave, y la intolerancia a la incertidumbre suele acompañarse de un marco legal más voluminoso e ineficiente y de menor calidad, que impide el libre flujo de conocimientos y su puesta en marcha (Hoefstede, 2001). Esta idea es compartida por Shane (1993, López y Vidal (2010) y Rinne et al. (2012) y se comprueba en el presente estudio.

Queda por analizar el efecto de la indulgencia. Las culturas indulgentes tienden a satisfacer las necesidades inmediatas y los deseos personales de sus miembros, y se caracterizan por la libre expresión de opiniones y por una elevada protección de los derechos humanos (Minkov y Hoefstede, 2011), lo que explica su relación positiva y significativa con la sustentabilidad social en este trabajo. En contraste, las sociedades que valoran la contención poseen normas sociales estrictas que regulan y desaconsejan la satisfacción de los impulsos. La indulgencia, según Hoefstede y Bond (1990), atiende algunos aspectos no cubiertos por las otras 5 dimensiones, pero vinculados a la literatura especializada en la felicidad. Las sociedades indulgentes tienden a disfrutar de la vida. Poseen una actitud optimista, dan mayor importancia al tiempo libre y al confort, así como a la calidad del medio ambiente, tal y como se comprueba en este trabajo. No obstante, uno de los problemas de esta característica social es el derroche, lo que podría explicar la correlación negativa débil que tiene con pragmatismo, aun cuando su vinculación con competitividad es robusta y positiva. Quizás por ello los resultados muestran que la indulgencia se potencia a través del pragmatismo, ya que sólo adquiere relevancia como motor de la sustentabilidad social y medioambiental si la sociedad posee una visión de largo plazo. Asimismo, la represión y la ausencia de esparcimiento parecen inhibir la formación de sociedades sanas, social y medioambientalmente hablando. Los polos positivos y negativos de esta dimensión explican por qué aun cuando las economías que se encuentran en el extremo indulgente, como México o Nigeria, han crecido más lentamente, en otros donde existe un mayor pragmatismo, parece darse una mayor competitividad, calidad del medioambiente y equidad social, como los Países Bajos. De igual forma que sucede con el individualismo, una vez alcanzado cierto nivel de desarrollo, si todo el mundo persigue intereses individuales, la economía crece menos rápidamente de lo que sería si al menos algunas personas trabajaran para propósitos colectivos. 
Existen también estudios ${ }^{23}$ que comprueban los peligros sociales que se derivan de esta característica cultural: un mayor desarrollo puede traer consigo una mayor indulgencia hacia sí mismo, la familia y el círculo social, a la vez que una mayor intolerancia a terceros. Encuestas aplicadas por distintos organismos ${ }^{24}$, muestran que las sociedades más avanzadas son menos tolerantes a cualquier desviación de aquello que consideran normal y aceptable y que amenace su modo de vida. Entre otras cosas, esto se traduce en castigos más severos, mayor rigor en las medidas para mantener el orden público, mayores restricciones al campo de acción de las personas e inclusive, a una mayor presencia de movimientos xenófobos. Esta tesis se ve reforzada con trabajos que confirman la existencia de una correlación positiva entre desigualdad del ingreso o la ausencia de un estado del bienestar y los índices delictivos, la informalidad, la fuga de cerebros y la emigración, así como el estancamiento económico ${ }^{25}$.

El análisis anterior sugiere que un modelo único de corte transversal es insuficiente para capturar el vínculo entre aspectos culturales como el individualismo y la indulgencia, en la formación de capital, así como su interacción, ya que parece cambiar según la competitividad del país. La imposibilidad de utilizar datos de panel al no contar con la información para un estudio de largo plazo de los cambios culturales endógenos contemplados en la Nueva Economía Institucional no exime, sin embargo, de intentar capturarlo. Por ello, se decide dividir la muestra total de países (97 países) según su nivel de competitividad, si bien el tamaño reducido de las submuestras obliga a no contemplar divisiones adicionales para países en transición, dejándolos en su categoría inicial ${ }^{26}$. De la muestra total, las submuestras son las siguientes: la etapa orientada a los factores de producción (23 países), la etapa con énfasis en la eficiencia (40 países) y la etapa impulsada por la innovación (34 países). El propósito de este ejercicio ha sido verificar si la significancia de los rasgos culturales se mantiene (robustez) y si sus coeficientes se modifican. Los resultados de estos ejercicios, presentados en los cuadros 2, 3 y 4, corroboran las observaciones intuitivas de párrafos anteriores.

La formación de capital físico depende positivamente del pragmatismo y se potencia conforme aumenta el mercado en sociedades avanzadas, mientras que en países de competitividad media, aun cuando el pragmatismo sigue siendo determinante, la penetración financiera se revela como necesaria para la inversión. En los países en vías de desarrollo, la formación de capital físico está a merced de la coyuntura económica y sujeta a la existencia de instituciones de calidad.

En países de elevada competitividad, el capital humano, bajo la forma de educación superior y capacitación, y dado un sistema adecuado de salud y educación primaria, depende positiva y significativamente de la eficiencia de los mercados laborales y de la alfabetización tecnológica. En esta fase, el capital intelectual y otros intangibles son la principal fuente de crecimiento económico y estos se crean y transmiten rápidamente gracias a las tecnologías de la información, lo que requiere de una alfabetización tecnológica generalizada (Lin y Edvinsson, 2008 y Labra y Sánchez, 2013). Al mismo tiempo, un mercado laboral eficiente permite retribuir económicamente la mayor productividad derivada de la formación de capital humano. Los rasgos culturales no parecen ejercer ningún papel significativo, si bien en países orientados a la eficiencia, el capital humano se ve mermado por la distancia del poder y es mayor cuando el mercado es más amplio. Finalmente, en países con menores niveles de

\footnotetext{
${ }^{23}$ Encuestas aplicadas por el Pew Research Center, http://www.pewsocialtrends.org/category/publications/2014/ y la encuesta World Value Survey, http://www.worldvaluessurvey.org/wvs.jsp.

${ }^{24}$ Véanse las encuestas del pie de página anterior..

${ }^{25}$ Véase por ejemplo Espinosa, Santiago, García y Hernández (2014).

${ }^{26}$ Esta simplificación no constituye un inconveniente ya que al haber utilizado un promedio del índice de casi 10 años, esta información queda capturada de forma indirecta.
} 
desarrollo cobra relevancia la alfabetización tecnológica y el individualismo para su formación. Al incluir alfabetización tecnológica en la regresión como variable instrumental para explicar la formación de capital humano, se evitan problemas de doble causalidad, ya que este último se utiliza como variable explicativa del avance tecnológico.

\begin{tabular}{|c|c|c|c|c|c|c|}
\hline \multicolumn{7}{|c|}{$\begin{array}{l}\text { CUADRO 2: PRUEBA DE HIPÓTESIS PARA LA MUESTRA DE PAÍSES EN } \\
\text { PRIMERA ETAPA DE DESARROLLO: LA ETAPA ORIENTADA A } \\
\text { FACTORES DE PRODUCCIÓN }\end{array}$} \\
\hline & $H_{0} a: K_{f}$ & $H_{0} b: K_{h}$ & $\mathrm{H}_{\mathrm{o}} \mathrm{c}: \mathrm{K}_{\mathrm{s} 1}$ & $\mathrm{H}_{0} \mathrm{~d}: \mathrm{K}_{\mathrm{s} 2}$ & $\mathrm{H}_{0} \mathrm{e}: \mathrm{K}_{\mathrm{n}}$ & $H_{0} \mathbf{f}: A$ \\
\hline$C$ & & $\begin{array}{c}\mathbf{- 0 . 8 2 3 8 *} \\
(-1.82)\end{array}$ & $\begin{array}{c}\mathbf{1 . 1 8 6 2}^{*} \\
(1.70)\end{array}$ & & & $\begin{array}{c}\mathbf{0 . 8 9 4 1}^{* * *} \\
(2.12)\end{array}$ \\
\hline$G C I$ & & & $\begin{array}{c}\mathbf{0 . 8 6 9 0} * * * * \\
(4.55)\end{array}$ & & & \\
\hline$D P$ & & & & $\begin{array}{c}-\mathbf{0 . 0 1 3 9} * * * * \\
(-3.53)\end{array}$ & & \\
\hline$I D V$ & & $\begin{array}{c}\mathbf{0 . 0 2 0 6} * * * * \\
(3.37)\end{array}$ & & & & \\
\hline$M A$ & & & $\begin{array}{l}-\mathbf{0 . 0 1 6 5}^{* *} \\
(-2.26)\end{array}$ & & & \\
\hline$A T$ & & $\begin{array}{c}\mathbf{1 . 2 0 9 2} * * * \\
(8.13)\end{array}$ & & & & \\
\hline$P G$ & & & & $\begin{array}{c}\mathbf{0 . 0 1 4 0}{ }^{* * * *} \\
(3.32)\end{array}$ & & \\
\hline$I D G$ & & & & $\begin{array}{c}\mathbf{0 . 0 1 2 5} * * \\
(2.05)\end{array}$ & & \\
\hline$E M$ & $\begin{array}{c}\mathbf{0 . 2 3 0 8 *}^{*} \\
(1.95)\end{array}$ & & & & & \\
\hline$R Q$ & & & & & $\begin{array}{c}\mathbf{0 . 4 0 5 6}^{*} \\
(2.05)\end{array}$ & \\
\hline$K_{h}$ & & & & $\begin{array}{c}\mathbf{0 . 8 9 3 3 K}_{\mathbf{h}} \text { **** } \\
(8.81)\end{array}$ & & $\begin{array}{c}\mathbf{0 . 2 7 6 6 * * *} \\
(2.81)\end{array}$ \\
\hline$K_{s 1}$ & $\begin{array}{c}\mathbf{0 . 5 1 8 8}^{* * * *} \\
(3.55)\end{array}$ & & & & $\begin{array}{c}\mathbf{0 . 5 7 6 5} * * \\
(2.69)\end{array}$ & $\begin{array}{c}\mathbf{0 . 3 1 5 6}^{* * *} \\
(2.66)\end{array}$ \\
\hline$R^{2}$ & 0.56 & 0.81 & 0.52 & 0.80 & 0.26 & 0.56 \\
\hline$R^{2} a j u s t a d a$ & 0.54 & 0.79 & 0.47 & 0.77 & 0.21 & 0.51 \\
\hline$F$ & na & na & $10.86^{* * *}$ & na & na & $12.60 * * *$ \\
\hline$n$ (países) & 23 & 23 & 23 & 23 & 18 & 23 \\
\hline
\end{tabular}




\section{CUADRO 3: PRUEBAS DE HIPÓTESIS PARA LA MUESTRA DE PAÍSES EN LA SEGUNDA ETAPA DE DESARROLLO: LA ETAPA ORIENTADA A LA EFICIENCIA}

\begin{tabular}{|c|c|c|c|c|c|c|}
\hline & $\mathbf{H}_{\mathbf{o}} \mathbf{a}: \mathbf{K}_{\mathrm{f}}$ & $\mathbf{H}_{\mathbf{o}} \mathbf{b}: \mathbf{K}_{\mathrm{h}}$ & $H_{0} c: K_{s 1}$ & $H_{0} d: K_{s 2}$ & $\mathbf{H}_{\mathbf{o}} \mathbf{e}: K_{n}$ & $\mathbf{H}_{\mathbf{0}} \mathbf{f}: \mathbf{A}$ \\
\hline GCI & & & $\begin{array}{c}1.0912 * * * \\
(16.01)\end{array}$ & & & \\
\hline$D P$ & & $\begin{array}{c}-\mathbf{0 . 0 1 2 4} * * \\
(-3.84)\end{array}$ & & $\begin{array}{c}-\mathbf{0 . 0 1 3 9} * * * \\
(-3.53)\end{array}$ & & \\
\hline$S P$ & & $\begin{array}{c}\mathbf{0 . 7 9 0 4} * * * \\
(15.40)\end{array}$ & & & & \\
\hline$M A$ & & & $\begin{array}{c}-\mathbf{0 . 0 0 7 1} * \\
(-1.95)\end{array}$ & & & \\
\hline$A I$ & & & $\begin{array}{c}\mathbf{- 0 . 0 0 5 5} * \\
(-1.90)\end{array}$ & & & \\
\hline$P G$ & $\begin{array}{c}\text { 0.0076* } \\
(1.83)\end{array}$ & & & $\begin{array}{c}\mathbf{0 . 0 1 1 4} * * * \\
(3.32)\end{array}$ & & \\
\hline$I D G$ & & & & $\begin{array}{c}\mathbf{0 . 0 1 2 5} * \\
(2.05)\end{array}$ & & \\
\hline$T M$ & & $\begin{array}{c}\mathbf{0 . 1 3 7 0} * * * \\
(2.89)\end{array}$ & & & & \\
\hline$E M$ & & & & & $\begin{array}{c}\mathbf{0 . 2 8 9 4} * * * \\
(3.82)\end{array}$ & \\
\hline$M F$ & $\begin{array}{c}\mathbf{0 . 8 3 4 4} * * * \\
(18.35)\end{array}$ & & & & & \\
\hline$K_{h}$ & & & & $\begin{array}{c}\mathbf{0 . 8 9 3 3}^{* * *} \\
(8.81)\end{array}$ & $\begin{array}{c}\mathbf{0 . 3 4 9 3} \text { **** } \\
(4.21)\end{array}$ & $\begin{array}{c}\mathbf{0 . 3 6 0 5} * * * \\
(5.60)\end{array}$ \\
\hline$K_{s I}$ & & & & & $\begin{array}{c}\mathbf{0 . 3 5 1 4} \mathbf{4}^{* * *} \\
(3.61)\end{array}$ & $\begin{array}{c}\mathbf{0 . 4 4 2 0} * * * \\
(6.43)\end{array}$ \\
\hline$R^{2}$ & 0.35 & 0.66 & 0.52 & 0.80 & 0.66 & 0.57 \\
\hline$R^{2} a j u s t a d a$ & 0.33 & 0.65 & 0.49 & 0.77 & 0.64 & 0.55 \\
\hline n (países) & 32 & 40 & 40 & 29 & 34 & 40 \\
\hline
\end{tabular}

Fuente: Elaboración propia con cifras de WEF (2014), Hofstede (1980, 1991, 1993, 2002 y 2008), Hofstede y Bond (1990), Hofstede y Arrindell (1998), Hofstede et al. (2008) y Behbudi et al. (2010).

El capital social formal de un país, representado por sus instituciones, se atrofia parcialmente en sociedades masculinas o con mayor distancia del poder, y crece conforme el país se desarrolla. Estas relaciones son robustas, ya que se mantienen significativas cualquiera que sea el nivel de competitividad del país. Lo mismo sucede con la sustentabilidad social, que se promueve en una nación con una elevada inversión en capital humano, pero también son determinantes rasgos culturales como la indulgencia y el pragmatismo para inducirla, así como la distancia del poder lo es para inhibirla. 


\section{CUADRO 4: PRUEBAS DE HIPÓTESIS PARA LA MUESTRA DE PAÍSES EN LA TERCERA ETAPA DE DESARROLLO: ETAPA ORIENTADA A LA INNOVACIÓN}

\begin{tabular}{|c|c|c|c|c|c|c|}
\hline & $\mathbf{H}_{\mathrm{o}} \mathrm{a}: \mathrm{K}_{\mathrm{f}}$ & $H_{o} b: K_{h}$ & $H_{0} c: K_{s 1}$ & $H_{0} d: K_{s 2}$ & $\mathbf{H}_{\mathbf{o}} \mathrm{e}: \mathbf{K}_{\mathrm{n}}$ & $H_{0} f: A$ \\
\hline$C$ & & $\begin{array}{c}-4.7183 * * * \\
(-3.83)\end{array}$ & & & & $\begin{array}{c}\mathbf{- 3 . 3 1 7 3} * * * \\
(-4.13)\end{array}$ \\
\hline$G C I$ & & & $\begin{array}{c}1.1329 * * * \\
(29.84)\end{array}$ & & & \\
\hline$D P$ & & & $\begin{array}{c}-\mathbf{0 . 0 0 7 9} * * \\
(-2.28)\end{array}$ & $\begin{array}{c}-\mathbf{0 . 0 1 3 9} * * * \\
(-3.53)\end{array}$ & & \\
\hline$S P$ & & $\begin{array}{c}1.2968 * * * \\
(5.59)\end{array}$ & & & & \\
\hline$M A$ & & & $\begin{array}{c}-\mathbf{0 . 0 0 6 0} * \\
(-1.96)\end{array}$ & & & \\
\hline$A T$ & & $\begin{array}{c}\mathbf{0 . 1 9 1 3} * \\
(1.74)\end{array}$ & & & & \\
\hline$P G$ & $\begin{array}{c}\mathbf{0 . 0 0 6 7} * \\
(1.71)\end{array}$ & & & $\begin{array}{c}\mathbf{0 . 0 1 4 0} * * * * \\
(3.32)\end{array}$ & & $\begin{array}{c}\mathbf{0 . 0 1 0 3}^{* * *} \\
(2.61)\end{array}$ \\
\hline$I D G$ & & & & $\begin{array}{c}\mathbf{0 . 0 1 2 5}^{*} \\
(2.05)\end{array}$ & & $\begin{array}{c}\mathbf{0 . 0 0 9 0} * \\
(1.68)\end{array}$ \\
\hline$T M$ & $\begin{array}{c}\mathbf{0 . 3 3 1 3}^{* * * *} \\
(4.06)\end{array}$ & & & & & \\
\hline$M F$ & $\begin{array}{c}\mathbf{0 . 7 1 8 1} * * * \\
(9.85)\end{array}$ & & & & & \\
\hline$M L$ & & $\begin{array}{c}\mathbf{0 . 1 7 5 6} * \\
(1.89)\end{array}$ & & & & \\
\hline$K_{h}$ & & & & $\begin{array}{c}\mathbf{0 . 8 9 3 3} * * * * \\
(8.81)\end{array}$ & $\begin{array}{c}\mathbf{0 . 2 7 9 7} * * \\
(2.38)\end{array}$ & $\begin{array}{c}1.2785 * * * \\
(7.64)\end{array}$ \\
\hline$K_{s 1}$ & & & & & $\begin{array}{c}\mathbf{0 . 5 3 8 4} * * * \\
(4.62)\end{array}$ & \\
\hline$A$ & & & & & $\begin{array}{c}\mathbf{0 . 2 5 8 6} \\
(2.21)\end{array}$ & \\
\hline$R^{2}$ & 0.51 & 0.80 & 0.74 & 0.80 & 0.84 & 0.77 \\
\hline$R^{2} a j u s t a d a$ & 0.48 & 0.79 & 0.72 & 0.77 & 0.83 & 0.75 \\
\hline$F$ & na & $40.99 * * *$ & na & na & na & $32.46 * * *$ \\
\hline$n$ (países) & 34 & 34 & 34 & 29 & 29 & 33 \\
\hline
\end{tabular}

Fuente: Elaboración propia con cifras de WEF (2014), Hofstede (1980, 1991, 1993, 2002 y 2008), Hofstede y Bond (1990), Hofstede y Arrindell (1998), Hofstede et al. (2008) y Behbudi et al. (2010).

La sustentabilidad medioambiental no parece depender directamente de un rasgo social en concreto. En países en desarrollo se promueve a través de un marco institucional sólido y conforme se cubren los requerimientos básicos de una sociedad. En países de competitividad media, si bien prevalece la importancia del marco institucional, cobra relevancia la educación para inducir al cuidado del medioambiente, pero sujeto a la coyuntura económica, En sociedades avanzadas, el capital social y el marco institucional son sus principales motores, y los factores coyunturales ceden su lugar a los avances tecnológicos y a la innovación. Este 
resultado es congruente con la curva ambiental de Kuznets. En la primera etapa de desarrollo planteada por el WEF, la degradación ambiental aumenta conforme lo hace la producción industrial con un uso intensivo de materias primas. En la medida en que aumenta el ingreso per cápita de estos países, también lo hace la demanda por recursos naturales. Una vez que dicha industrialización alcanza su máximo, inicia un proceso de terciarización gradual de la economía, que incide favorablemente en la calidad ambiental, tanto por la menor contaminación, como por el uso menos intensivo de materias primas, energía y recursos naturales, así como por el arbitraje regulador que hace que los procesos productivos más contaminantes se desplacen a países con leyes ambientales más laxas (Angulo, 2008), por lo que aunque en el ámbito nacional la degradación ambiental tiene forma de $\mathrm{U}$ invertida en países avanzados, no la tiene en el ámbito global puesto que no todos los países alcanzarán el tramo de pendiente negativa de la curva de Kuznets. La calidad ambiental sería entonces un lujo, donde los países más desarrollados demandan una mayor calidad medioambiental y tienen los recursos para proveerla, mediante avances tecnológicos e instituciones de calidad, entre otros ${ }^{27}$. Esta se potencia en sociedades más equitativas.

La innovación, por su parte, depende de la formación de capital humano y se desarrolla más en sociedades a la vez indulgentes y pragmáticas, una vez que el país adquiere cierta calidad institucional. Los intangibles presentan una estrecha relación con el desempeño económico en la era del conocimiento, sobre todo cuando se gestionan adecuadamente mediante política pública de largo plazo que tome en cuenta el carácter acumulativo y dinámico del conocimiento (Lin y Edvinsson, 2008). En países de competitividad media y baja, lo importante es el marco legal, la educación superior y la capacitación.

La formación de capital humano y la sustentabilidad social se inhiben en una estructura social jerárquica, lo que impide un mayor desarrollo de los países. Asimismo, las sociedades masculinas, competitivas, con una mayor brecha entre roles y la búsqueda del éxito material como prioridad, merman la sustentabilidad social de una nación. Si este resultado se combina con el encontrado para individualismo, que cede su protagonismo a la indulgencia como promotor de la competitividad conforme el país se desarrolla, se confirma la importancia de la calidad de vida y de la empatía para garantizar el crecimiento. En el mismo sentido, los resultados comprueban la intuición económica (Minkov y Hofstede, 2011): la indulgencia no afecta significativamente al crecimiento de un país en desarrollo, pero lo potencia en naciones de mayor industrialización a través de la formación de capital humano y el avance tecnológico. De hecho, la indulgencia es central para la sustentabilidad social, requisito para el bienestar y la paz social a largo plazo. Es más, las regresiones muestran cómo el pragmatismo, considerado por varios autores como el motor del crecimiento, sólo es significativo en países de elevada competitividad si son indulgentes.

\section{CONCLUSIONES}

Este trabajo ha investigado la posible relación entre los rasgos culturales de un grupo seleccionado de países y los principales determinantes del crecimiento económico: la formación de capital físico, humano, social, natural y el avance tecnológico. Los resultados obtenidos demuestran que la cultura influye en ellos a través de distintos canales, y que aun cuando esta relación no sea estable en el tiempo o varíe de acuerdo al grado de competitividad

\footnotetext{
${ }^{27} \mathrm{Al}$ incluir avance tecnológico en la regresión como variable instrumental y no el índice de competitividad para explicar la sustentabilidad medioambiental, se evitan problemas de doble causalidad. Empíricamente, esta relación sólo se cumple a nivel nacional para algunos contaminantes concretos, tales como el dióxido de sulfuro, el dióxido de azufre o las partículas suspendidas (Stern, 2004).
} 
alcanzado por un país, su signo positivo o negativo, según el rasgo cultural del que se trate, es consistente entre países y en distintas etapas de competitividad. Todas las dimensiones culturales presentaron los signos esperados y fueron significativas en la formación de alguna modalidad de capital y/o en el avance tecnológico de los países. Destacan particularmente el pragmatismo, el individualismo y el igualitarismo y en menor medida la indulgencia y la tolerancia a la incertidumbre. Aun cuando los datos sólo permitían un análisis de corte transversal, este resultaba insuficiente para capturar la naturaleza dinámica de la relación entre cultura y crecimiento, o dicho de otra forma. Por ello, se subdividió la muestra total de países según su nivel de competitividad para verificar su robustez y enriquecer los resultados obtenidos. Si Hoefstede encontró que sólo el pragmatismo es determinante en el crecimiento económico, quizás sea porque al ser la variable cultural más robusta en sus resultados, pudiera ser la única en capturar el vínculo entre cultura y crecimiento para el periodo donde hay cifras disponibles, ya que el crecimiento es más lento en países industrializados, conforme lo prevén las teorías de convergencia económica. El uso de indicadores de competitividad promedio de la última década, en sustitución a tasas de crecimiento económico, resuelve este problema.

Las implicaciones de esta investigación son amplias. En primer lugar, ha demostrado que los valores culturales son lo suficientemente descriptivos de una economía como para inferir sobre sus niveles de competitividad relativa. Esto no quiere decir que la acumulación de capital se rija únicamente por variables culturales: existen otros determinantes, además de las variables de control consideradas en este trabajo, cuya exclusión llevaría a una subespecificación de cualquier modelo de competitividad y crecimiento.

En segundo lugar, este trabajo muestra cómo la vinculación de cultura y desempeño económico varía según el grado de competitividad alcanzado por el país, confirma el impacto negativo de la distancia del poder y en menor medida de la aversión a la incertidumbre en la conformación de capital y cuestiona el papel del individualismo como motor de crecimiento, particularmente para las naciones más industrializadas. Este estudio también destaca la importancia de la indulgencia en el crecimiento. En la medida en que una persona o país logran afianzar su situación económica, pueden darse el lujo de tener mayor cantidad y calidad de ocio y esparcimiento, deportes, viajes, actividades culturales y alimentación de calidad, y que la preocupación por el ahorro deje de ser tan relevante. En el mundo industrializado, la sensación de bienestar en las personas se vincula a la indulgencia. De la misma forma, una sociedad que restringe el goce de la vida en aras de una mayor riqueza, virtud $\mathrm{u}$ orden, se relaciona negativamente con el empoderamiento y la competitividad, aun cuando a corto plazo parece favorecer la eficiencia de los mercados. Las sociedades avanzadas con bajos niveles de indulgencia tienden a ser más pesimistas y ansiosas, lo que desalienta la creatividad y el espíritu empresarial. La indulgencia no sólo es significativa por sí sola para explicar la formación de capital en países de alta competitividad, sino que además, potencia el efecto del pragmatismo y la innovación.

Como señala el WEF (2014), la inestabilidad mundial y el desbordamiento de los fenómenos migratorios evidenciados recientemente alertan sobre cómo un crecimiento económico excluyente, inequitativo y desequilibrado puede socavar su continuidad intergeneracionalmente. Si se percibe de forma generalizada una distribución desigual de los beneficios económicos, y esta desigualdad lleva al descontento e inestabilidad social, más allá de consideraciones éticas o de bienestar social, tanto el proceso de crecimiento como la capacidad de los individuos para contribuir y beneficiarse de este se verán severamente afectados. Por ejemplo, se demuestra en este estudio que la formación de capital humano se promueve en sociedades donde la eficiencia del mercado laboral permite retribuir una mayor productividad, sea mediante mayores salarios o mejores oportunidades de promoción 
profesional. De esta forma, el concepto de competitividad sostenible cobra relevancia, ya no sólo como objetivo, sino como insumo macroeconómico, al obligar a la existencia de un conjunto de instituciones, políticas y factores que hacen que una nación sea productiva a largo plazo, al tiempo que garantiza la sostenibilidad social y ambiental, elementos que hacen a las sociedades prósperas y que garantizan un crecimiento de calidad. Lo que se pretende medir es, no sólo si un país tiene el potencial de crecer en el mediano y largo plazo, sino también si dicho proceso da lugar al tipo de sociedad en el que la mayoría quiere vivir.

La fiabilidad de las cifras, así como la diversidad de países incluidos en el análisis, proveen de información relevante tanto en el ámbito teórico, como para la evidencia empírica y los negocios. Su principal valor, desde el punto de vista de sus aportaciones y originalidad, es que aun con sus limitaciones, es de los pocos trabajos sobre estos temas que intentan ser de carácter multidisciplinar, incorporando elementos de distintas áreas de conocimiento, que interactúan para identificar determinantes no convencionales del desempeño económico.

La definición estrecha del capital social que subyace en este trabajo, aun cuando permite la cuantificación de tópicos poco explorados, se constituye como una severa limitación que no debe subestimarse. Por una parte, esta definición restringe el estudio al campo nacional, sin contemplar las diferencias existentes dentro de un mismo país o región, y sólo infiere sobre los cambios intertemporales implícitos en estas dimensiones. Asimismo, el análisis también se limita al número de países para los que las puntuaciones de WEF y de Hoefstede están disponibles, lo que provoca que varias naciones del Medio Oriente y de África sean excluidas. No obstante, se intenta subsanar esta deficiencia mediante la subdivisión de la muestra según la etapa de desarrollo y la inclusión de la sustentabilidad social como parte del mismo.

Cabe destacar que ningún trabajo de este tipo está exento de problemas debido precisamente a esas diferencias culturales, de naturaleza subjetiva, que intenta medir. La complejidad de medición de los intangibles crece conforme lo hace el sujeto de estudio, por lo que las herramientas de corte microeconómico son difíciles de aplicar en un entorno más amplio, independientemente del modelo elegido (Käpylä et al., 2012). Aun así, contribuye a la estandarización del estudio de estos tópicos, tan relevantes como poco explorados. $\mathrm{Su}$ principal aportación es que obliga a establecer prioridades de análisis, al considerar las fuentes de información y sus límites, de forma esquemática y mediante una comparación internacional. Ello coadyuva a un diseño gradual de indicadores conforme se profundiza en una metodología más rigurosa y conforme el sistema de información evoluciona para poder alimentarla. Este enfoque combate la tendencia a que la discusión y evaluación de un método se centren en su rigor estadístico y no en la importancia de los supuestos y parámetros elegidos, a menudo más relevantes y con mayor significado interpretativo.

Otra limitación de este estudio tiene que ver con el carácter unidireccional establecido entre cultura y crecimiento. Es indispensable un análisis de los efectos del cambio cultural intergeneracional, de la diversidad cultural dentro de cada nación e incluso de la fusión cultural, en las instituciones formales, en la cultura empresarial así como en los distintos indicadores de productividad en un escenario de largo plazo. Johnson y Lenartowicz (1998) consideran que las correspondencias halladas por Hofstede (1980) entre individualismo y PIB per cápita y más tarde entre pragmatismo y crecimiento económico (Franke et al., 1991), no demuestra sin embargo un sentido definido de causalidad, tal y como lo reconocen Hoefstede y Bond. Este trabajo comparte esta percepción, aun cuando se ha verificado que dicha endogeneidad no alcanza a invalidar los resultados obtenidos. Un estudio en profundidad sobre la naturaleza de estos vínculos es una de las líneas de investigación más prometedoras que se derivan de este documento. Sería enriquecedor identificar, con mayor precisión, los canales a través de los cuales los rasgos culturales fomentan o inhiben la productividad y el 
crecimiento, mediante un análisis de los subíndices que componen a cada uno de los indicadores del WEF utilizados. De esta manera, podrían diseñarse programas de formación de capital humano, tanto en instituciones formales de educación superior como en distintos organismos de educación técnica, capacitación e incubadoras de negocio, que tengan en cuenta las características culturales específicas del entorno en el que se van a implementar.

Existen diversos canales a través de los cuales la cultura incide en el desempeño de un país que no se exploran en este estudio. En concreto, los rasgos culturales determinan, no sólo la creación de instituciones sino su operatividad, y no sólo la competitividad sino el desarrollo.

Otra posible extensión de este trabajo surge de la observación de que los valores culturales afectan al comportamiento individual y colectivo de acuerdo al contexto y a la propia cultura. Así por ejemplo, si algunos países anglosajones han sido capaces de lograr un desempeño económico ejemplar con su estilo de trabajo individualista y su énfasis en el éxito material, otros países como Japón también lo han logrado con un modelo distinto con énfasis en la educación, el compromiso y la lealtad entre instituciones e individuos. Asimismo, países del sudeste asiático poseen rasgos de las dos culturas, potenciando los aspectos positivos de ambas. Este tema merece una exploración a profundidad.

Finalmente, se sugiere el uso de ecuaciones estructurales para un mayor conocimiento de las relaciones entre variables culturales y entre estas y los indicadores de competitividad. La utilidad de estos modelos radica en que proveen una visión global del fenómeno de estudio, permiten incorporar variables no medibles directamente y no requieren corroborar las relaciones causales.

\section{BIBLIOGRAFÍA}

Altman, M. (2001): "Culture, human agency, and economic theory: Culture as a determinant of material welfare", Journal of Socio-Economics 30, pp.379-391.

Alzati, F. (1997): The political economy of growth in modern Mexico, Tesis doctoral, Harvard University.

Angulo, A (2008): "Relación entre crecimiento económico y medio ambiente: la U ambiental de Kuznets", Desarrollo local sostenible, 3(8), pp. 1-8

Arndt, HW. (1978): The Rise and Fall of Economic Growth, Melbourne: Longman Cheshire.

Azariadis, C. y Drazen, A. (1990): “Threshold Externalities in Economic Development”, Quarterly Journal of Economics 105, pp. 501-526

Barro, R y Sala-i-Martin, X (1995): Economic Growth, McGraw-Hill, NY.

Becker, G (1998): Accounting for Tastes, Cambridge: Harvard University Press.

Becker G. (1996): Preferences and Values, In Becker Gary (ed.), Accounting for Taste, Harvard University Press, Cambridge.

Becker, G. (1976): The Economic Approach to Human Behavior,. In Becker, G, The Economic Approach to Human Behavior, University of Chicago Press, pp. 3 - 14.

Behbudi, D., Mamipour, S. y Karami, A. (2010): "Natural resource abundance, human capital and economic growth in petroleum exporting countries", Journal of Economic Development, 35.

Benzaquen, L., Del Carpio, A. Zegarra L. y Valdivia, C. (2010): "Índice regional de competitividad para un país", Revista de la CEPAL 102, Diciembre, pp. 69 - 86.

De la Fuente, A. y Jimeno J. F. (1999): Crecimiento Económico: Teoría, Aplicaciones y Evidencia Empírica, Instituto de Análisis Económico (CSIC) y Universidad de Alcalá, Apuntes de Clase. 
Blum, Ulrich y Dudley, L. (2001): "Religion and economic growth: Was Weber right?", Journal of Evolutionary Economics, 11, pp. 207-230.

Clark, T. (1990): "International marketing and national character: A review and proposal for an integrative theory", Journal of Marketing, 54(4), pp. 66-79.

Clark, C (1940): Las Condiciones del Progreso Económico, Editorial Alianza, 1971.

Coleman, J. S. (1990): Foundations of Social Theory, Harvard University Press, Cambridge.

Cooper, C. (1982): "Culture consequences: international differences in work related values", Journal of Occupational behavior (April), pp. $202-204$.

Craig, C. y Douglas, S. (2006): "Beyond national culture: Implications of cultural dynamics for consumer research", International Marketing Review, 23(3), pp. 322-342.

Dawar, N. y Parker, P. (1994): "Marketing universals: Consumers' use of brand name, price, physical appearance, and retailer reputation as signals of product quality", Journal of Marketing, 58(2), pp. 8195.

Diamond, J. (1998): Armas, Gérmenes y Acero. La Sociedad Humana y sus Decisiones, Ed. Debate Pensamiento, Madrid.

Domar, E. (1946): “Capital Expansion, Rate of Growth, and Employment”, Econometrica, vol. 14, n. 2, pp. 137-147.

Edvinsson, L. (2003): The intellectual capital of nations. In C. Holsapple (Ed.), Handbook of knowledge management 1. knowledge matters, Berlin: Springer, pp. 153-164.

Edvinsson, L. (1999): "The intellectual capital of nations: the future wealth creation", Journal of Human Resource and Accounting, vol. 4, $\mathrm{n}^{\circ}$ 1, Spring, p. 21-23

Espinosa, M, Santiago, M., García J., y Hernández, O. (2014): "Remesas, desigualdad y pobreza: Un caso de estudio en el estado de Oaxaca", Estudios Fronterizos, nueva época, vol. 15, núm. 29, enerojunio de 2014, pp. 125-141.

Faria, J. y León Ledesma, M. (2004): Cultural Heritage and Growth, School of Social Sciences, University of Texas at Dallas, USA.

Franke, R, Hofstede, G. y Bond M, (1991):" Cultural roots of economic performance: a research note”, Strategic Management Journal, vol. 12, pp. 165-173.

Hill, Ch. (2009): International business: competing in the global marketplace, McGraw-Hill/Irwin, USA.

Hofstede, G. (2011): "Dimensionalizing Cultures: The Hofstede Model in Context", Online Readings in Psychology and Culture, 2(1). http://dx.doi.org/10.9707/2307-0919.1014

Hofstede, G. Hofstede, G.J. y Minkov, M. (2010): Cultures and Organizations: Software of the Mind, Third Edition McGraw-Hill; USA, $3^{\text {th }}$ edition.

Hofstede, G. (2003): Culture's Consequences: Comparing Values, Behaviors, Institutions and Organizations across Nations ( $2^{\text {nd }}$ edition), USA Sage Publications.

Hofstede, G. y Arrindell, W, (1998): Masculinity and Femininity: The Taboo Dimension of National Cultures, Sage Publications, Inc., London, pp. 119 - 120.

Hofstede, G. (1991): Cultures and Organizations: Software of the Mind, McGraw-Hill, London.

Hofstede, G. (1980): "Motivation, Leadership, and Organization: Do American Theories Apply Abroad?", Organizational Dynamics, vol. 9 n 1, Summer 1980, pp. 42-63.

Hofstede, G. y Bond, M. (1990): “The Confucius Connection: From Cultural Roots to Economic Growth", Organizational Dynamics, vol. 16 n 4, Spring 1988, pp. 4-21. 
Hofstede, G., Hofstede, G. J., Minkov, M. y Vinken, H. (2008): Values Survey Module 2008 Manual, BV, Recuperado de: http://stuwww.uvt.nl/\%7Ecsmeets/ManualVSM08.doc

House, R. J., Hanges, P. J., Javidan, M., Dorfman, P. W., y Gupta, V. (2004): Leadership, Culture, and Organizations: The GLOBE Study of 62 Societies, Thousand Oaks: Sage Publications.

Hsu, S, Woodside,A. y Marshall R. (2012): Critical Tests of Multiple Theories of Cultures Consequences Comparing the Usefulness of Models by Hofstede, Inglehart and Baker, Schwartz, Steenkamp, and GDP and Distance for Explaining Overseas Tourism Behavior.

Disponible en: http://www.polyu.edu.hk/htm/iast/newsletter/pdf/v23i102b_woodside_cri.pdf

Inglehart, R. y Welzel, C. (2005): Modernization, Cultural Change, and Democracy: The Human Development Sequence, New York: Cambridge University Press.

Inglehart, R. (1997): Modernization and postmodernization, Princeton University Press.

Jaén, I., Fernández-Serrano J. y Liñán, F. (2013):" Valores culturales, nivel de ingresos y actividad emprendedora", Revista de Economía Mundial, 35, pp. 21-45.

Javidan, M, Johnson, J. y Lenartowicz, T. (1998): "Culture, Freedom and Economic Growth: Do Cultural Values Explain Economic Growth?”, Journal of World Business, 33(4): pp. 332-356.

Johnson, J. y Lenartowicz, T. (1998): "Culture, freedom and economic growth: do cultural values explain economic growth?”, Journal of World Business 33/4 1998.

Kaldor, D. (1963): Capital Accumulation and Economic Growth. In Proceedings of a Conference Held by the International Economics Association editado por Friedrich Lutz, A and Douglas C. Haugue, eds., London, Macmillan.

Kápyla, J., Kujansivu, P. y Lónnqvist, A. (2012): "National intellectual capital performance: a strategic approach", Journal of Intellectual Capital, vol. 13, n 3, pp. 343-362.

Labra and Sánchez (2013):"National intellectual capital assessment models: a literature review", Journal of Intellectual Capital, vol. 14 Iss: 4, pp. 582 - 607.

Lin, C.Y.Y. y Edvinsson, L. (2008): "National intellectual capital: comparison of the Nordic Countries", Journal of intellectual Capital, vol. $9, \mathrm{n}^{\circ} 4$.

Lin, C. Y.Y. y Edvinsson, L. (2011): National intellectual capital: A comparison of 40 countries. New York, USA: Springer Lynn (1991). Citado en Hofstede et al. (1998). Masculinity and Femininity: The Taboo Dimension of National Cultures, USA Sage Publications, Inc., 1998, pp. 119 - 120.

López Duarte, C. y Vidal Suárez, M. (2010): "La influencia de la distancia cultural en el proceso de crecimiento internacional. ¿Una cuestión de paradigma? Factores culturales e internacionalización de la empresa y la economía", Sept-Oct 2010. ICE, n. ${ }^{\circ} 856$, pp. 21-40.

Makarov, P. (2010): "Intellectual capital as an indicator of a sustainable development", Journal of Sustainable Development, 3(3), pp. 85-90.

McSweeney, B. (2002): "Hofstede's model of national cultural differences and their consequences: A triumph of faith - A failure of analysis", Human Relations, 55(1), pp. 89-118.

Minkov, M, Hofstede, G. y Blagoev, V. (2013): "The boundaries of culture: Do questions about societal norms reveal cultural differences?", Journal of Cross-Cultural Psychology, vol.44 no.7, pp. 1094-1106.

Minkov, M. y Hofstede, G (2011):"The evolution of Hofstede's doctrine", Cross Cultural Management: An International Journal, vol. 18, $\mathrm{n}^{\circ}$ 1, pp. 10-20.

North, D. (1990): Institutions, Institutional Change and Economic Performance, Cambridge University Press, Cambridge 
OCDE (2003): Human Capital Investment. An International Comparison, Centre for Educational Research and Innovation, Paris, France.

Porter, M. E. (1990): The Competitive Advantage of Nations, Free Press, New York.

Romer, D. (2000): Advanced Macroeconomics, 2a Edición, USA. Editorial Mc. Graw Hill.

Ros, M. y Gouveia V.(2003): "Psicología social de los valores humanos: desarrollos teóricos, metodológicos y aplicados", Revista Interamericana de Psicología, vol. 37, n¹, pp. 171 -176.

Rostow, W. (1960): The Stages of Economic Growth, a Non- Communist Manifesto, Cambridge: University Press.

Rinne, T, Steel, D. y Fairweather, J. (2012): Hofstede and Shane Revisited "The Role of Power Distance and Individualism in National-Level Innovation Success", Cross-Cultural Research, May, vol. $46, \mathrm{n}^{\circ} .2$ pp. 91-108.

Shane, S. (1993): "Cultural Influences on National Rates of Innovation", Journal of Business Venturing, 8(1), pp. 59-73.

Schumpeter, J. (1934): The Theory of Economic Development, Cambridge, Harvard University Press

Schwartz, S. H. (2006): Value orientations: Measurement, antecedents and consequences across nations. In Jowell, R., Roberts, C., Fitzgerald, R. \& Eva, G. (Eds.), Measuring attitudes crossnationally - lessons from the European Social Survey, London: Sage.

Smith, A. (1776): An Inquiry into the Nature and Causes of the Wealth of Nations, 1 (1 Ed.), W. Google Books, London.

Solow, R. (1956): “A Contribution to the Theory of Economic Growth", Quarterly Journal of Economics, 70, pp. 65-94

Steenkamp, J. (2001): "The role of national culture in international marketing research", International Marketing Review, 18(1), pp. 30-44.

Stern, D (2004): "The rise and fall of the environmental Kutznets curve", World Development 32(8), pp. 1419-1439.

Swan, T. W. (1956): "Economic growth and capital accumulation", Economic Record (Wiley) 32 (2), November, pp. 334-361.

Taras, V. (2010): Catalogue of Instruments for Measuring Culture. Recuperado en 2010 por Hsu et al., (2010) de: http://vtaras.com/Culture_Survey_Catalogue

Tortella,G. y Núñez, C. (2014): El desarrollo de la España contemporánea. Historia económica de los siglos XIX y XX, Alianza Editorial S. A. Madrid.

Tylor, E. (1924), Primitive Culture, 2 vols, 7th Ed, New York: Brentano's (orig. 1871).

Yeganeh, H. (2013): "An investigation into the cultural and religious determinants of national competitiveness", Competitiveness Review, vol. 23 Issue 1, pp. 23-40.

WEF, World Economic Forum (2014): The Global Competitiveness Report 2014 - 2015. Disponible en: http://www.weforum.org/reports/global-competitiveness-report-2014-2015. 